\title{
Factors Influencing Public Attitudes towards COVID-19 Vaccination: A Scoping Review Informed by the Socio-Ecological Model
}

\author{
Ghadir Fakhri Al-Jayyousi ${ }^{1, * \mathbb{D}}$, Mohamed Abdelhady Mabrouk Sherbash ${ }^{1}$, Lamees Abdullah Mohammed Ali ${ }^{1}$ (D), \\ Asmaa El-Heneidy ${ }^{2}$, Nour Waleed Zuhair Alhussaini ${ }^{1}$, Manar Elsheikh Abdelrahman Elhassan ${ }^{1}$ D \\ and Maisa Ayman Nazzal ${ }^{3}$ \\ 1 Department of Public Health, College of Health Sciences, QU Health, Qatar University, \\ Doha P.O. Box 2713, Qatar; msherbash@qu.edu.qa (M.A.M.S.); la1605823@qu.edu.qa (L.A.M.A.); \\ nwaleed@qu.edu.qa (N.W.Z.A.); melhassan@qu.edu.qa (M.E.A.E.) \\ 2 School of Medicine and Dentistry and Menzies Health Institute Queensland, Griffith University, Gold Coast \\ Campus, Southport, QLD 4222, Australia; a.el-heneidy@griffith.edu.au \\ 3 Department of Pharmacy, Faculty of Medicine and Health Sciences, An Najah National University, \\ Nablus 44839, West Bank, Palestine; s11525766@stu.najah.edu \\ * Correspondence: g.aljayyousi@qu.edu.qa
}

check for updates

Citation: Al-Jayyousi, G.F.; Sherbash, M.A.M.; Ali, L.A.M.; El-Heneidy, A.; Alhussaini, N.W.Z;; Elhassan, M.E.A.; Nazzal, M.A. Factors Influencing Public Attitudes towards COVID-19 Vaccination: A Scoping Review Informed by the Socio-Ecological Model. Vaccines 2021, 9, 548. https:/ / doi.org/10.3390/vaccines 9060548

Received: 18 April 2021

Accepted: 19 May 2021

Published: 24 May 2021

Publisher's Note: MDPI stays neutral with regard to jurisdictional claims in published maps and institutional affiliations.

Copyright: (c) 2021 by the authors. Licensee MDPI, Basel, Switzerland. This article is an open access article distributed under the terms and conditions of the Creative Commons Attribution (CC BY) license (https:// creativecommons.org/licenses/by/ $4.0 /)$

\begin{abstract}
Major hindrances to getting a COVID-19 vaccine include vaccine hesitancy, skepticism, refusal, and anti-vaccine movements. Several studies have been conducted on attitudes of the public towards COVID-19 vaccines and the potential influencing factors. The purpose of this scoping review is to summarize the data available on the various factors influencing public attitudes towards COVID-19 vaccination. This scoping review was conducted according to the Preferred Reporting Items for Systematic Reviews and Meta-Analyses extension for Scoping Reviews (PRISMA-ScR) Statement. PubMed, Embase, Web of Science, and Cochrane Central were searched without restrictions to reclaim all publications on the factors that shape individuals' attitudes towards COVID-19 vaccines from 1 January 2020 to 15 February 2021. Fifty studies were included. The scoping review revealed that the factors influencing public attitudes towards COVID-19 vaccines were embedded within the different levels of the socio-ecological model. These factors included the sociodemographic characteristics of the individuals, individual factors, social and organizational factors. In addition, certain characteristics of COVID-19 vaccines themselves influenced public attitudes towards accepting the vaccines. Understanding various population needs and the factors shaping public attitudes towards the vaccines would support planning for evidence-based multilevel interventions in order to enhance global vaccine uptake.
\end{abstract}

Keywords: COVID-19; vaccine; hesitancy; acceptance; refusal; willingness; ecological model; scoping review

\section{Introduction}

Coronavirus disease 2019 (COVID-19) is a contagious and pathogenic viral infection caused by severe acute respiratory syndrome coronavirus 2 (SARS-CoV-2), a specific type of coronavirus that was first discovered in Wuhan, China [1]. It was declared a global pandemic by the World Health Organization on the 11 March 2020. The pandemic caused by COVID-19 has infected more than 125 million people and killed at least 2.5 million globally and is becoming a leading cause of death [2]. This virus has become a major concern around the globe, having so many consequences on the healthcare system and economy and instilling fear in communities [3,4]. The main mode of transmission is through droplets, direct contact with infected patients; it can also be transmitted through fomites, by touching contaminated surfaces or objects [5]. People who are at increased risk of getting severe infection include the elderly and those who have chronic diseases [6]. 
Although many efforts have been dedicated to the implementation of suppression strategies including travel bans, partial/full lockdown, contact tracing, and social distancing, the transmission of the virus is more likely to rebound when these strategies are lifted [7]. Consequently, for a long-term approach to combating this epidemic, the development and use of vaccines is essential [8].

Vaccination stimulates the immune system to develop antibodies to fight a specific infectious agent in the body [9]. They have been used to eliminate and significantly decrease morbidity and mortality associated with different infectious diseases [10] by providing benefit to those who get vaccinated and also protecting communities through reducing transmission of the disease [10]. Via herd immunity, a high uptake of COVID19 vaccines can also help protect people who cannot get a vaccine such as those with compromised immune systems and young children [11]. Getting efficacious results from a vaccine does not solely rely on accessibility/uptake, but also depends upon the public's acceptance and willingness to get vaccinated [11]. Other major hindrances to getting a vaccine include vaccine hesitancy, skepticism, refusal, and anti-vaccine movements [12]. In 2019, vaccine hesitancy was identified as one of the ten challenges to global health [13], and this concern has grown throughout the COVID-19 pandemic [14]. While prior studies looked at predictors of vaccine acceptance and uptake, it is worth noting that emergencyreleased vaccines differ from established vaccinations in many aspects [15], and newer vaccines are usually met with greater skepticism [16].

Several studies have been conducted on attitudes of the public towards COVID-19 vaccines and potential influencing factors $[15,17]$. It is imperative to investigate the different factors influencing attitudes and perceptions of people related to COVID-19 vaccines. Vaccine refusal has a variety of causes which differ depending on regional, cultural, and social factors [18]. Understanding different vaccine attitudes is particularly significant as diverse vaccine refusal strategies that address the needs of different groups can be developed [19]. In the light of the COVID-19 pandemic, there is an urgent need for a more comprehensive and detailed understanding of attitudes toward vaccines and the factors affecting vaccine intention in order to adjust public health messages as appropriate [20]. Therefore, in this scoping review, we sought to rapidly explore the determinants influencing public attitudes with respect to COVID-19 vaccines and provide a more comprehensive and nuanced understanding of how these factors shape certain perspectives and behaviors.

\section{Materials and Methods}

This scoping review was conducted according to the Preferred Reporting Items for Systematic Reviews and Meta-Analyses extension for Scoping Reviews (PRISMA-ScR) Statement (see Appendix A) [21]. The study has no written or published a priori protocol. Our research question was as follows: what are the factors influencing public attitudes with respect to COVID-19 vaccines? After identification of the research question, we identified relevant studies, selected the studies, charted the data, and collated, summarized, and reported the findings.

\subsection{Information Sources}

PubMed (National Library of Medicine, Bethesda, MD, USA), Embase (Elsevier, Amsterdam, The Netherlands), Web of Science (Clarivate Analytics, Philadelphia, PA, USA), and Cochrane Central (Cochrane, London, UK) were searched without restrictions to reclaim all publications on the individual factors, sociocultural factors, and environmental factors that shape an individual's decision (attitude) towards COVID-19 vaccines from 1 January 2020 to 15 February 2021. Table 1 describes the search strategies used to collect published articles from the databases. Reference lists of the selected articles were also searched for articles that might have been missed in the online database search. 
Table 1. Search strategies.

\begin{tabular}{|c|c|}
\hline Search & Search Term \\
\hline$\# 1$ & “Corona” OR “SARS-CoV-2" OR “COVID 19” \\
\hline \#2 & "Vaccine" OR “Vaccination" \\
\hline \#3 & $\begin{array}{c}\text { “Acceptance" OR “Agreement” OR “Willingness” OR } \\
\text { “Refusal” OR “Resistance” OR “Confidence” OR } \\
\text { "Hesitancy" OR “Antivaxx" OR “Antivaxxers" OR } \\
\text { "Antivaccine" OR “Anti-vaccine" }\end{array}$ \\
\hline$\# 4$ & $\# 1, \# 2$, and \#3 \\
\hline
\end{tabular}

\subsection{Eligibility Criteria}

In this scoping review, all the articles published between 1 January 2020 to 15 February 2021 about the factors that shape public attitudes towards COVID-19 vaccines were included. The articles selected were only in English, with human subjects aged 16 years and above, excluding healthcare workers who are obligated to get a vaccine. Articles were only included in English since it was the major language of the available articles in the databases at the time this search was conducted.

Two authors independently screened abstracts and citations retrieved from the search and each author (out of the seven authors) was given a specific number of articles to assess full texts of the relevant records to be included in the review. When dealing with duplicates, the most recent version of the article with the largest sample size was included. During the study selection and assessment process, the first author was responsible for resolving any disagreements and final evaluation.

\subsection{Data Charting Process}

The relevant data were abstracted from the eligible articles in pre-structured data charting forms (Excel (Microsoft Corporation, Redmond, WA, USA) and Word (Microsoft Corporation, Redmond, WA, USA) documents). The following information was included: the first author of the study, publication year, study design, population, study setting, mean age of the participants (in years), sample size, attitude towards COVID-19 vaccines, and the various factors shaping these public attitudes. Descriptive statistics (percentages) were reported for some studies to reflect the rate of positive or negative attitudes towards the vaccines. The final column in the table reported percentages to reflect prevalence of the factors shaping the attitudes, and for some studies, the $p$-value was reported to reflect the significant association between the factors and public attitudes towards the vaccines.

\subsection{Synthesis of Results}

Description of the scope of literature was presented in tables according to the key levels of the socio-ecological model [22] which showed how a health determinant (public attitudes towards COVID-19 vaccines) can be influenced by the various factors embedded in different levels. In our review, we summarized and clustered the factors that influenced public attitudes towards COVID-19 vaccines into sociodemographic characteristics, individual factors, and social and organizational factors. The final set of factors that were related to the specific characteristics of COVID-19 vaccines were summarized and reported in a separate table.

\subsection{Data Analysis}

Outcome data related to the factors that influence public attitudes towards COVID-19 vaccines were summarized and clustered into the different levels of the socio-ecological model. Meta-analysis was not performed due to heterogeneity in the contextual and environmental factors including the healthcare system in the countries of the selected studies in our review. 


\section{Results}

In total, 331 records were retrieved from the electronic database search. The remaining records, after removing the duplicates, amounted to 274 records. After screening the titles and abstracts, 185 were excluded; the remaining 89 full-text articles were assessed for eligibility, and 50 studies were reserved for this review. The PRISMA diagram illustrates the study selection process and shows the reasons for exclusion for other studies (Figure 1).
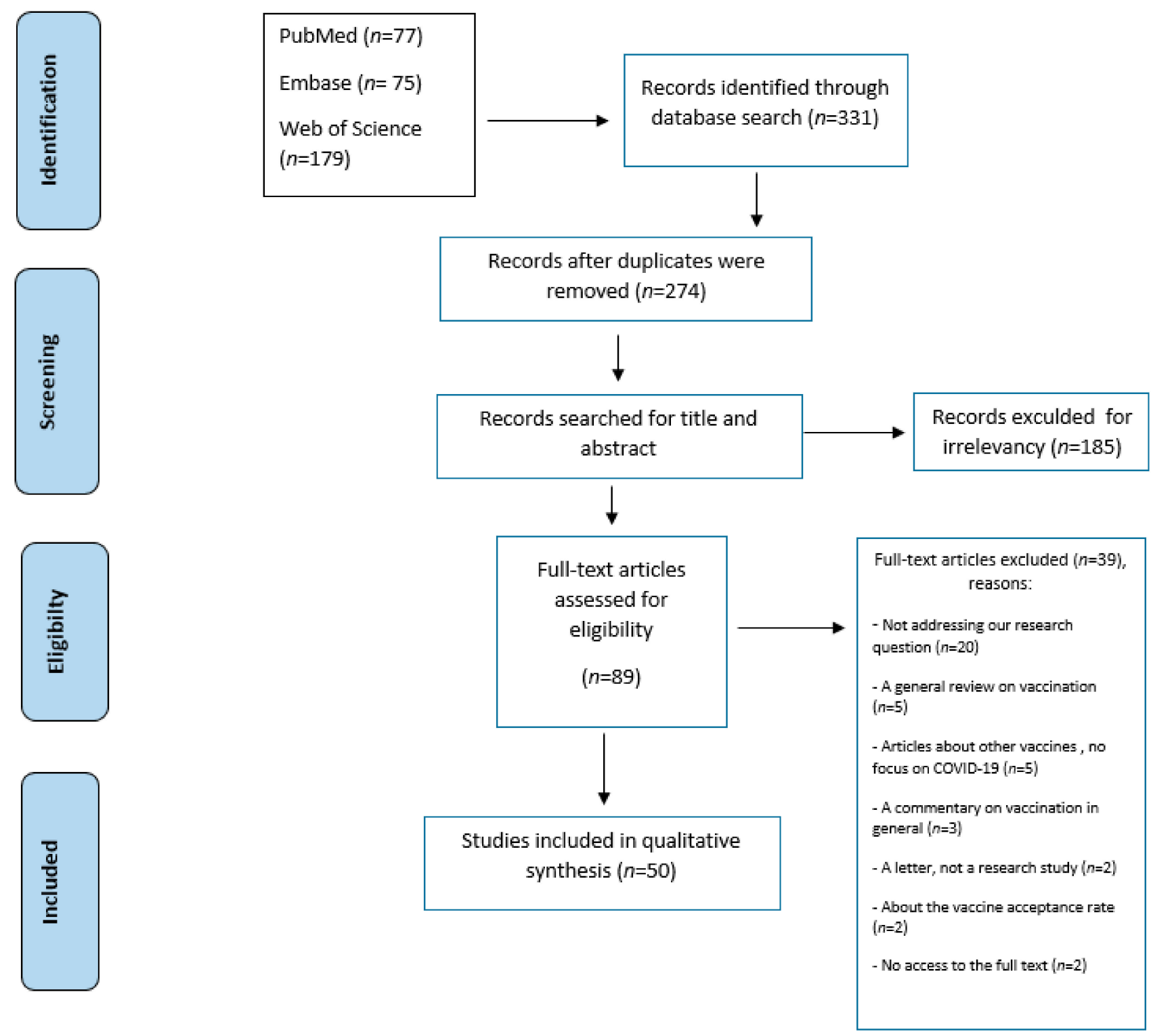

Figure 1. PRISMA flow diagram of study selection.

\subsection{Study Characteristics}

The studies were divided by design into one experimental study [23], 39 cross-sectional studies [24-62], five literature reviews [63-67], one systematic review [68], one randomized controlled trial [69], one longitudinal survey with two experiments [70], one Gallup panel [71], one media analysis study through a proposed novel behavioral dynamics model SRS/I (susceptible-reading-susceptible/immune) for the microblogging platform Weibo on social media [72], and one conference paper [73]. The majority of these crosssectional studies reported their findings from one country, one study reported data from 19 countries [25], another study conducted legal analysis for several countries [40], and 
others reported findings from two or three countries from Europe, America, Canada, and the Middle East $[29,49,59]$. The sample size of the reviewed studies ranged from 101 participants in an RCT study [69] to 13,426 participants in a survey conducted in 19 countries [25].

An online (web-based) survey was the most common data collection method applied in the cross-sectional studies in our review, of which:

- Some studies reported using social media platforms including Facebook, Twitter, and LinkedIn to collect data $[26,38,41,62,72]$;

- Some studies reported using specific platforms to upload surveys including crowdsourcing platform Amazon Mechanical Turk (mTurk, Amazon, Seattle, WA, USA) [32,61], computer-assisted web interviewing (CAWI) and computer-assisted telephone interviewing (CATI) [34,53], and Qualtrics (Seattle, WA, USA) [47,55];

- One study used an online questionnaire to examine public attitudes towards COVID-19 vaccines in three phases: pre-lockdown, during lockdown, and post lockdown with different participants in every phase [41], and another study conducted two-wave follow-up online surveys [55];

- One study used an online survey with a semi-structured questionnaire following the snowball sampling technique [60];

- One study used open-ended questions to ask about the factors influencing attitudes towards vaccines [47];

- One study used an Internet survey and telephone interviews [35];

- One study used a face-to-face-administered questionnaire [37].

The target populations in the majority of the reviewed articles were adults from the general public. The youngest participants were recruited in a study from Italy in which the age ranged from 15 to 85 years [53], and another study was conducted among parents and guardians aged 16 years and above who reported living in England with a child aged 18 months or under [26]. Only three studies were conducted among populations with specific demographics such as the working population in Hong Kong (HK), China [52], people with respiratory chronic diseases and older adults aged 65 and above $[47,55]$, and the black American community in an RCT study [69].

The reported vaccine acceptance rate in the reviewed articles ranged from $29.4 \%$ to $86 \%$ [23,24,26,45-49,51,72]. A study from the Middle East [49] reported a low rate of acceptance among the public from Jordan, Kuwait, and Saudi Arabia (29.4\%), while a higher acceptance of $53.1 \%$ was reported in a study from Kuwait [48]. On the other hand, $86 \%$ of people in the UK expressed their willingness to receive a vaccine [47]. The information on the baseline characteristics of the selected studies can be found in Table 2. 
Table 2. Baseline characteristics of the selected studies.

\begin{tabular}{|c|c|c|c|c|c|c|}
\hline $\begin{array}{c}\text { First Author/Year of } \\
\text { Publication }\end{array}$ & Study Design & Study Setting & Population & Sample Size & $\begin{array}{l}\text { Mean Age } \\
\text { (in Years) }\end{array}$ & $\begin{array}{c}\text { Attitudes towards } \\
\text { COVID-19 Vaccines }\end{array}$ \\
\hline Chen, T.E. 2021 [23] & Experimental design & Online experiment & Chinese adults & 413 & $\begin{array}{l}\text { Aged between } 18 \text { and } 60 \\
(\mathrm{M}=24.70, \mathrm{SD}=9.55)\end{array}$ & $\begin{array}{l}\text { Attitudes towards } \\
\text { COVID-19 vaccination } \\
\text { scores were highly } \\
\text { favorable and the intention } \\
\text { to get vaccinated was high }\end{array}$ \\
\hline Chen, M.S. 2020 [24] & Cross-sectional & Online questionnaire & Chinese adults & 3195 & Majority aged 18-44 & $\begin{array}{l}83.8 \% \text { were willing to } \\
\text { receive a COVID-19 vaccine, } \\
13.6 \% \text { were unsure }\end{array}$ \\
\hline Lazarus, J.V. 2020 [25] & Cross-sectional & & 19 countries (global) & 13,426 & Majority aged 25-54 & $\begin{array}{l}46.8 \% \text { completely agreed to } \\
\text { accept a COVID-19 vaccine }\end{array}$ \\
\hline Bell, S. 2020 [26] & Cross-sectional survey & Online social media strategy & $\begin{array}{l}\text { Parents and guardians (aged } \\
\text { 16+ years) who reported living } \\
\text { in England with a child aged } \\
18 \text { months or under }\end{array}$ & 1190 & 33 & $\begin{array}{l}\text { Acceptability of a future } \\
\text { COVID-19 vaccine }\end{array}$ \\
\hline Coustasse, A. 2020 [63] & Review & & US population & & & $\begin{array}{l}50 \% \text { willing to get a vaccine } \\
\text { in study } 1 \\
65 \% \text { willing to get a vaccine } \\
\text { in study } 2\end{array}$ \\
\hline Robles, A.S. 2020 [73] & Conference paper & & Nevada & & & Vaccine acceptance \\
\hline Wang, J. $2020[28]$ & Online cross-sectional survey & Public & China & 2058 & Adults & $\begin{array}{l}\text { Willingness to accept a } \\
\text { vaccine }\end{array}$ \\
\hline Lin, C. 2021 [68] & Systematic review & & & 126 surveys & & $\begin{array}{l}\text { Vaccine intention and } \\
\text { acceptance }\end{array}$ \\
\hline Murphy, J. 2021 [29] & Cross-sectional survey & Online survey & $\begin{array}{l}\text { General adult populations of } \\
\text { Ireland and the United } \\
\text { Kingdom }\end{array}$ & 3066 & $\begin{array}{c}\text { Mostly 55-64 (Ireland) and } \\
45-54 \text { (UK) }\end{array}$ & $\begin{array}{c}\text { COVID-19 vaccine } \\
\text { hesitancy and resistance, } \\
\text { including psychological } \\
\text { indicators }\end{array}$ \\
\hline Akarsu, B. 2020 [30] & $\begin{array}{l}\text { Cross-sectional web-based } \\
\text { survey }\end{array}$ & Public & General public in Turkey & 759 & $32.41 \pm 9.92$ & Vaccination acceptance \\
\hline Seale, H. 2021 [31] & National cross-sectional survey & Online survey & $\begin{array}{c}\text { Australian adults (18 years and } \\
\text { older) }\end{array}$ & 1420 & $\begin{array}{c}\text { Mostly } \\
30-49\end{array}$ & $\begin{array}{c}\text { Perceptions and behaviors } \\
\text { towards a future COVID-19 } \\
\text { vaccine }\end{array}$ \\
\hline
\end{tabular}


Table 2. Cont.

\begin{tabular}{|c|c|c|c|c|c|c|}
\hline $\begin{array}{l}\text { First Author/Year of } \\
\text { Publication }\end{array}$ & Study Design & Study Setting & Population & Sample Size & $\begin{array}{l}\text { Mean Age } \\
\text { (in Years) }\end{array}$ & $\begin{array}{l}\text { Attitudes towards } \\
\text { COVID-19 Vaccines }\end{array}$ \\
\hline Hursh, S.R. 2020 [32] & Cross-sectional survey & $\begin{array}{c}\text { Online using crowdsourcing } \\
\text { platform Amazon Mechanical } \\
\text { Turk (mTurk) }\end{array}$ & $\begin{array}{c}\text { Participants from the United } \\
\text { States }\end{array}$ & 534 & 41.9 & $\begin{array}{l}\text { Evaluation of COVID-19 } \\
\text { vaccine demand }\end{array}$ \\
\hline Biasio, L.R. 2020 [33] & Online survey & Online study & Italian adults & 885 & Majority aged 31-50 & $\begin{array}{c}\text { Perceptions about getting a } \\
\text { vaccine }\end{array}$ \\
\hline Kourlaba, G. 2021 [34] & Cross-sectional survey & $\begin{array}{l}\text { Computer-assisted telephone } \\
\text { interviewing (CATI) and } \\
\text { computer-assisted web } \\
\text { interviewing (CAWI) }\end{array}$ & Adult Greeks & 1004 & $41.7(17.7)$ & $\begin{array}{l}\text { Willingness to receive a } \\
\text { COVID-19 vaccine }\end{array}$ \\
\hline Fisher, K. 2020 [35] & Cross-sectional survey & $\begin{array}{l}\text { Internet survey and telephone } \\
\text { interviews }\end{array}$ & US general public & 1000 & Adults & Vaccine hesitancy \\
\hline Guidry, J.P.D. 2021 [36] & Cross-sectional survey & Online survey & US adults & 788 & 45.9 & $\begin{array}{l}\text { Willingness to get a } \\
\text { COVID-19 vaccine }\end{array}$ \\
\hline Jung, H. 2020 [70] & $\begin{array}{c}\text { Longitudinal survey, two } \\
\text { experiments }\end{array}$ & & United states & $\begin{array}{c}\text { Survey: } 2490 \\
\text { Experiment: } 800\end{array}$ & & Vaccination intention \\
\hline Popa, G.L. 2020 [37] & $\begin{array}{c}\text { Face-to-face cross-sectional } \\
\text { survey }\end{array}$ & Public & Romanian respondents & 1647 & Median age, 37 & Vaccination acceptance \\
\hline Prati, G. 2021 [39] & Online survey & Online study & General public in Italy & 624 & Between 18 and 72 years & $\begin{array}{l}\text { Willingness to accept a } \\
\text { vaccine }\end{array}$ \\
\hline Marco-Franco, J.L. 2021 [40] & Cross-sectional, legal analysis & & Several countries & & & Vaccine hesitancy \\
\hline Caserotti, M. 2021 [41] & $\begin{array}{l}\text { Cross-sectional online } \\
\text { questionnaire } \\
\text { (pre-lockdown, during } \\
\text { lockdown, and post-lockdown) } \\
\text { Note: "different participants } \\
\text { every phase" }\end{array}$ & $\begin{array}{l}\text { Various institutional and } \\
\text { personal social channels } \\
\text { related to the research team }\end{array}$ & Italian residents & 2267 & $25-65$ & $\begin{array}{c}\text { The percentage of people } \\
\text { who accepted a vaccine was } \\
\text { high during the lockdown }\end{array}$ \\
\hline Bogart, L.M. 2021 [69] & $\mathrm{RCT}$ & Community-based & Black Americans & 101 & $50.3(11.5)$ & $\begin{array}{l}\text { COVID-19 mistrust beliefs, } \\
\text { COVID-19 vaccine or } \\
\text { treatment hesitancy }\end{array}$ \\
\hline
\end{tabular}


Table 2. Cont.

\begin{tabular}{|c|c|c|c|c|c|c|}
\hline $\begin{array}{l}\text { First Author/Year of } \\
\text { Publication }\end{array}$ & Study Design & Study Setting & Population & Sample Size & $\begin{array}{l}\text { Mean Age } \\
\text { (in Years) }\end{array}$ & $\begin{array}{l}\text { Attitudes towards } \\
\text { COVID-19 Vaccines }\end{array}$ \\
\hline Alley, S.J. 2021 [42] & Repeated cross-sectional survey & Online & Australian adults & 575 & Mostly over 55 years & $\begin{array}{l}\text { Willingness to get } \\
\text { vaccinated against } \\
\text { COVID-19 }\end{array}$ \\
\hline Puri, N. 2020 [64] & Review & Social media & & & & Vaccine hesitancy \\
\hline Reiter, P.L. 2020 [43] & Online survey & Online study & US general public & 2006 & $>18$ & $\begin{array}{l}\text { Willingness to accept a } \\
\text { vaccine }\end{array}$ \\
\hline Feleszko, W. 2021 [44] & $\begin{array}{l}\text { Poland, an online omnibus } \\
\text { survey tool }\end{array}$ & Public & Poland public & 1066 & $18-65$ & $\begin{array}{l}\text { 37\% supported COVID-19 } \\
\text { vaccination }\end{array}$ \\
\hline Danchin, M. 2020 [65] & Review & & & & & Vaccine refusal \\
\hline Harapan, H. 2020 [45] & Cross-sectional online survey & Online study & General population in Indonesia & 1359 & $\begin{array}{l}\text { More than half of the } \\
\text { respondents were aged } \\
21-30 \text { years }\end{array}$ & $\begin{array}{l}50 \text { or } 95 \% \text { effective } \\
\text { COVID-19 vaccine }\end{array}$ \\
\hline Lin, Y. 2020 [46] & $\begin{array}{l}\text { Nationwide cross-sectional } \\
\text { self-administered online survey }\end{array}$ & Public & China & 3541 & Adults & $\begin{array}{l}83.5 \% \text { said yes to the intent } \\
\text { to get a vaccine }\end{array}$ \\
\hline Williams, L. 2020 [47] & $\begin{array}{l}\text { Cross-sectional study with } \\
\text { open-ended questions about the } \\
\text { factors }\end{array}$ & Online & $\begin{array}{l}\text { Older adults and people with } \\
\text { respiratory chronic diseases }\end{array}$ & 527 & $\begin{array}{l}\text { Older sample with adults } \\
\text { aged } 65\end{array}$ & $\begin{array}{c}86 \% \text { of respondents wanted } \\
\text { to receive a COVID-19 } \\
\text { vaccine }\end{array}$ \\
\hline Alqudeimat, Y.2021 [48] & Web-based cross-sectional study & $\begin{array}{l}\text { Public (adults living in } \\
\text { Kuwait) }\end{array}$ & Kuwait & 2368 & $>21$ & $\begin{array}{l}\text { Participants were willing to } \\
\text { accept a COVID-19 vaccine } \\
\text { once available (53.1\%) }\end{array}$ \\
\hline Sallam, M. 2021 [49] & Cross-sectional online survey & Online study & $\begin{array}{l}\text { Jordan, Kuwait, and Saudi } \\
\text { Arabia }\end{array}$ & 3414 & & Vaccine acceptance $(29.4 \%)$ \\
\hline Wong, L.P. 2020 [50] & Cross-sectional online survey & Online survey & Malaysian general public & 1159 & Adults & $\begin{array}{l}\text { Willingness to accept a } \\
\text { vaccine }\end{array}$ \\
\hline Nguyen, K.H. 2020 [51] & $\begin{array}{l}\text { CDC-conducted household } \\
\text { panel surveys }\end{array}$ & Internet survey & US general public & & Adults & $\begin{array}{l}\text { Willingness to accept a } \\
\text { vaccine }\end{array}$ \\
\hline
\end{tabular}


Table 2. Cont.

\begin{tabular}{|c|c|c|c|c|c|c|}
\hline $\begin{array}{c}\text { First Author/Year of } \\
\text { Publication }\end{array}$ & Study Design & Study Setting & Population & Sample Size & $\begin{array}{l}\text { Mean Age } \\
\text { (in Years) }\end{array}$ & $\begin{array}{l}\text { Attitudes towards } \\
\text { COVID-19 Vaccines }\end{array}$ \\
\hline Wang, K.L. 2021 [52] & Cross-sectional & Online questionnaire & $\begin{array}{l}\text { Working population in Hong } \\
\text { Kong (HK), China }\end{array}$ & 1196 & Majority aged $40-49$ & $\begin{array}{l}\text { Standardized rate of vaccine } \\
\text { acceptance in the first } \\
\text { survey was } 44.2 \% \text { and } 34.8 \% \\
\text { in the second survey }\end{array}$ \\
\hline Largent, E.A. 2020 [71] & Gallup panel & Online study & US adults & 2730 & Not available & $\begin{array}{c}\text { Acceptability of COVID-19 } \\
\text { vaccines }\end{array}$ \\
\hline LaVecchia, K. 2020 [53] & National cross-sectional survey & $\begin{array}{l}\text { Online using computer- } \\
\text { assisted web interviews } \\
\text { (CAWI) }\end{array}$ & Italians aged $15-85$ years & 1055 & 33.2 & $\begin{array}{l}\text { Attitudes towards a } \\
\text { potential } \\
\text { COVID-19 vaccine }\end{array}$ \\
\hline Ward, J.K. 2020 [54] & Cross-sectional survey & Online & $\begin{array}{l}\text { French } \\
\text { population of } 18 \text { years of age }\end{array}$ & 5018 & Mostly less than 35 & $\begin{array}{l}\text { Attitudes to a future } \\
\text { COVID-19 vaccine }\end{array}$ \\
\hline Romer, D. 2020 [55] & Two-wave follow-up surveys & Online using Qualtrics & US general population & 1050 & Mostly 60+ & $\begin{array}{c}\text { Intention to get vaccinated } \\
\text { against COVID-19 }\end{array}$ \\
\hline Sherman, S.M. 2020 [56] & Cross-sectional online survey & Online study & UK adults & 1500 & $46 \pm 15.8$ & Vaccination intention \\
\hline McCaffery, K.J. 2020 [57] & $\begin{array}{l}\text { National cross-sectional } \\
\text { community online survey }\end{array}$ & & $\begin{array}{l}\text { Australian general public, } \\
\text { adults aged over } 18 \text { years }\end{array}$ & 4362 & $42.6 \pm 17.4$ & Vaccine hesitancy \\
\hline Pogue, K. 2020 [58] & Cross-sectional survey & Public online survey & US respondents & 316 & & COVID-19 vaccine intention \\
\hline Taylor, S. 2020 [59] & Cross-sectional survey & Public Internet-based study & $\begin{array}{l}\text { American and } \\
\text { Canadian adults }\end{array}$ & 3674 & $53 \pm 15$ & Vaccination hesitancy \\
\hline Reuben, R.C. 2020 [60] & $\begin{array}{l}\text { Cross-sectional online survey } \\
\text { with a semi-structured } \\
\text { questionnaire using a snowball } \\
\text { sampling technique }\end{array}$ & Public & North-central Nigeria & 589 & $80.6 \%$ were $18-39$ years & $\begin{array}{l}29 \% \text { accepted to take a } \\
\text { vaccine }\end{array}$ \\
\hline Corpuz, R. 2020 [61] & $\begin{array}{l}\text { Cross-sectional Amazon's } \\
\text { Mechanical Turk (MTurk), an } \\
\text { online survey tool }\end{array}$ & Online study & US general public & 209 & $\begin{array}{l}\text { Mean age } \mathrm{M}=33.4 \text { years } \\
\qquad(\mathrm{SD}=11.4)\end{array}$ & $\begin{array}{l}\text { Endorsement of the } \\
\text { vaccines }\end{array}$ \\
\hline Bertin, P. 2020 [62] & $\begin{array}{l}\text { Two cross-sectional studies. An } \\
\text { online questionnaire was } \\
\text { disseminated by the authors on } \\
\text { Facebook, Twitter, and Linkedin }\end{array}$ & Online studies & General public in France & 396 & $\begin{array}{c}\mathrm{M}_{\mathrm{age}}=26.1, \mathrm{SD}=10.3 \\
\min =18, \max =70\end{array}$ & $\begin{array}{l}\text { Negatively predicted } \\
\text { participants intention to get } \\
\text { the vaccine }\end{array}$ \\
\hline Ling, R. 2020 [66] & Review & Social media & & & & Anti-vaccination \\
\hline Dube, E. 2020 [67] & Review & & & & & Vaccine hesitancy \\
\hline
\end{tabular}




\subsection{Sociodemographic Characteristics Shaping Public Attitudes towards COVID-19 Vaccines}

Twenty-eight studies depicted the sociodemographic factors associated with public attitudes (Table 3). Age $[23,25,27,29,31,34-36,38,51-53,63,65,73]$, educational level [23-25,27, $30,35,36,42,47,49-51,63,65,68,71,73]$, gender $[24,25,28-32,34,38,48,49,51,52,65,68]$, race $[26$, $29,35,36,47,51,63,68,71,73]$, and income status $[24-26,29,47,50,51,63,65,68]$ were the most common factors reported. White individuals older than 25 years who have a high education level and high-income status were more likely to report positive attitudes towards the vaccines. The majority of the reviewed studies reported that men were more willing to accept a vaccine than women $[28-30,38,48,49]$. Only one study in the USA reported higher willingness among men [32].

Table 3. Sociodemographic characteristics associated with hesitancy or acceptance of COVID-19 vaccines.

\begin{tabular}{|c|c|c|}
\hline First Author & $\begin{array}{c}\text { Hesitancy }(-) \text { or Acceptance }(+) \text { of } \\
\text { COVID-19 Vaccines }\end{array}$ & $\begin{array}{l}\text { Sociodemographics Shaping Public } \\
\text { Attitudes towards COVID-19 Vaccines }\end{array}$ \\
\hline Chen, T.E. 2021 [23] & + & $\begin{array}{l}\text { - High education level } \\
\text { - Increasing age }\end{array}$ \\
\hline Chen, M.S. 2020 [24] & + & $\begin{array}{l}\text { - Male gender } \\
\text { - High income } \\
\text { - Education level } \\
\text { - Han nationality }\end{array}$ \\
\hline \multirow[t]{2}{*}{ Lazarus, J.V. 2020 [25] } & + & $\begin{array}{l}\text { - Age older than } 25 \\
\text { - Male gender } \\
\text { - High income } \\
\text { - High education level }\end{array}$ \\
\hline & - & - Sick people or sick family members \\
\hline Bell, S. 2020 [26] & - & $\begin{array}{l}\text { - Black, Asian, Chinese, mixed, or other } \\
\text { ethnicity } \\
\text { - Low income }\end{array}$ \\
\hline Coustasse, A. 2020 [63] & + & $\begin{array}{l}\text { - } 60 \text { years and older } \\
\text { - Non-Hispanic Whites } \\
\text { - High education level } \\
\text { - High income }\end{array}$ \\
\hline Al-Mohaithef, M. 2020 [27] & - & $\begin{array}{l}\text { - Older age } \\
\text { - Being married } \\
\text { - High education level } \\
\text { - Non-Saudi } \\
\text { - Employed in the government sector }\end{array}$ \\
\hline Robles, A.S. 2020 [73] & + & $\begin{array}{l}\text { - Age } \\
\text { - Ethnicity } \\
\text { - Chronic disease } \\
\text { - Education level } \\
\text { - Employment status } \\
\text { - Country }\end{array}$ \\
\hline Wang, J. 2020 [28] & + & $\begin{array}{l}\text { - Male gender } \\
\text { - Being married }\end{array}$ \\
\hline Lin, C. 2021 [68] & + & $\begin{array}{l}\text { - College degrees } \\
\text { - Income, } \\
\text { - Insurance } \\
\text { - Living in rural or larger areas } \\
\text { - Gender } \\
\text { - Race }\end{array}$ \\
\hline
\end{tabular}


Table 3. Cont

\begin{tabular}{|c|c|c|}
\hline First Author & $\begin{array}{c}\text { Hesitancy }(-) \text { or Acceptance }(+) \text { of } \\
\text { COVID-19 Vaccines }\end{array}$ & $\begin{array}{l}\text { Sociodemographics Shaping Public } \\
\text { Attitudes towards COVID-19 Vaccines }\end{array}$ \\
\hline Murphy, J. 2021 [29] & - & $\begin{array}{l}\text { Irish sample, vaccine-hesitant } \\
\text { - Female gender } \\
\text { - Aged between } 35 \text { and } 44 \text { years } \\
\text { - No mental health problem } \\
\text { Irish sample, vaccine-resistant } \\
\text { - Aged 35-44 years } \\
\text { - Residing in a city } \\
\text { - Non-Irish ethnicity } \\
\text { - Lower income } \\
\text { - Underlying health condition } \\
\text { UK sample, vaccine-hesitant } \\
\text { - Female gender } \\
\text { - Younger than } 65 \\
\text { UK sample, vaccine-resistant } \\
\text { - Younger age } \\
\text { - More likely to reside in a suburb } \\
\text { - In the three lowest income brackets } \\
\text { - Being pregnant }\end{array}$ \\
\hline & - & $\begin{array}{l}\text { - Female gender } \\
\text { - Unemployed }\end{array}$ \\
\hline Akarsu, B. 2020 [30] & + & $\begin{array}{l}\text { - Have SSI or private health insurance } \\
\text { - Have children } \\
\text { - Those who were thinking about getting } \\
\text { their child a COVID-19 vaccine were more } \\
\text { willing to get vaccinated } \\
\text { - High level of education }\end{array}$ \\
\hline Seale, H. 2021 [31] & + & $\begin{array}{l}\text { - Female gender } \\
\text { - Aged } 70 \text { years and above } \\
\text { - Reporting chronic disease } \\
\text { - Holding private health insurance }\end{array}$ \\
\hline Hursh, S.R. 2020 [32] & - & - Male gender \\
\hline Kourlaba, G. 2021 [34] & + & $\begin{array}{l}\text { - Aged > } 65 \text { years old } \\
\text { - Those belonging to vulnerable groups or } \\
\text { members of their household belonging to } \\
\text { vulnerable groups }\end{array}$ \\
\hline Fisher, K. 2020 [35] & - & $\begin{array}{l}\text { - Young age } \\
\text { - Black race } \\
\text { - Low educational attainment }\end{array}$ \\
\hline Guidry, J.P.D. 2021 [36] & + & $\begin{array}{l}\text { - Education } \\
\text { - Having insurance } \\
\text { - Age } \\
\text { - Race/ethnicity }\end{array}$ \\
\hline Detoc, M. 2020 [38] & + & $\begin{array}{l}\text { - Older age, } \\
\text { - Male gender }\end{array}$ \\
\hline Alley, S.J. 2021 [42] & - & $\begin{array}{l}\text { - Low education } \\
\text { - Female gender }\end{array}$ \\
\hline Danchin, M. 2020 [65] & - & $\begin{array}{l}\text { - Low education } \\
\text { - Low income } \\
\text { - Potentially more prone to infectious diseases } \\
\text { - Women aged }<35 \text { years } \\
\text { - People aged }>75 \text { years who are at a higher } \\
\text { risk of disease from COVID-19 }\end{array}$ \\
\hline
\end{tabular}


Table 3. Cont.

\begin{tabular}{|c|c|c|}
\hline First Author & $\begin{array}{c}\text { Hesitancy (-) or Acceptance (+) of } \\
\text { COVID-19 Vaccines }\end{array}$ & $\begin{array}{l}\text { Sociodemographics Shaping Public } \\
\text { Attitudes towards COVID-19 Vaccines }\end{array}$ \\
\hline Lin, Y. 2020 [46] & + & - Self-employed and service sector workers \\
\hline Williams, L. 2020 [47] & + & $\begin{array}{l}\text { - White ethnicity } \\
\text { - High education level } \\
\text { - High income } \\
\text { - High-risk/shielding }\end{array}$ \\
\hline Alqudeimat, Y. 2021 [48] & + & - Male gender \\
\hline Sallam, M. 2021 [49] & + & $\begin{array}{l}\text { - Male gender } \\
\text { - High education levels } \\
\text { - History of chronic disease }\end{array}$ \\
\hline Wong, L.P. 2020 [50] & + & $\begin{array}{l}\text { - Higher education levels } \\
\text { - Professional and managerial occupations } \\
\text { - High income }\end{array}$ \\
\hline Nguyen, K.H. 2020 [51] & - & $\begin{array}{l}\text { - Young adults } \\
\text { - Female gender } \\
\text { - Non-Hispanic Black (Black) persons } \\
\text { - Adults living in nonmetropolitan areas } \\
\text { - Adults with lower educational attainment } \\
\text { - Low income } \\
\text { - No health insurance }\end{array}$ \\
\hline Wang, K.L. 2021 [52] & + & $\begin{array}{l}\text { - Young age } \\
\text { - Male gender } \\
\text { - Being married }\end{array}$ \\
\hline Largent, E.A. 2020 [71] & + & $\begin{array}{l}\text { - Non-Black respondents more likely to get } \\
\text { vaccinated } \\
\text { - Respondents with a bachelor's degree or } \\
\text { higher }\end{array}$ \\
\hline LaVecchia, K. 2020 [53] & + & $\begin{array}{l}\text { - Age above } 55 \\
\text { - Professionals, managers, teachers, and } \\
\text { manual workers }\end{array}$ \\
\hline
\end{tabular}

Moreover, other factors were included, such as health condition, people with chronic diseases [29,31,49,65,73], occupation status [27,30,46,50,53,73], marital status [27,28,52], place of residence $[29,51,68,73]$, women being pregnant [29] or having children [30], and those who have health insurance or not $[30,31,36,51]$. The reviewed articles showed that individuals who had chronic diseases, were employed, married with children, and had health insurance were more likely to report acceptance of COVID-19 vaccines.

\subsection{Individual Factors Shaping Public Attitudes towards COVID-19 Vaccines}

Several individual factors influencing public attitudes were reported in thirty-six studies (Table 4). Personal beliefs with regard to vaccines and COVID-19 [30,32,34,35,49,55-57,62,66,68,70], health literacy $[33,65]$, knowledge $[34,37,57,68]$, lack of trust in governments and companies producing the vaccines $[25,35,37,39,59]$, perceived susceptibility and risk perception towards COVID-19 and side effects of the vaccines [24,28,36,38,45-48,56], social [61,70], religious [37], and political views [29,32,54,61,71], level of anxiety of getting infected [30], fear $[24,30,37,38]$ and worries $[39,59]$, confidence in academic institutions and producing companies [24,60], preference towards natural immunity [40], previous experience with flu vaccines or other vaccines $[28,30,52,56,58]$, likelihood of infection and severity of the disease $[41,43,48,58]$ were all considered as individual factors in this scoping review. 
Table 4. Individual factors associated with hesitancy or acceptance of COVID-19 vaccines.

\begin{tabular}{|c|c|c|}
\hline First Author & $\begin{array}{c}\text { Hesitancy (-) or Acceptance }(+) \text { of } \\
\text { COVID-19 Vaccines }\end{array}$ & $\begin{array}{l}\text { Individual Factors Shaping Public } \\
\text { Attitudes towards COVID-19 Vaccines }\end{array}$ \\
\hline Chen, M.S. 2020 [24] & + & $\begin{array}{l}\text { - Confidence, satisfaction, and worries about } \\
\text { risks } \\
\text { - Attention to relevant COVID-19 information } \\
\text { - Perceived views of the severity of } \\
\text { COVID-19 disease } \\
\text { - Degree of concern regarding the COVID-19 } \\
\text { pandemic }\end{array}$ \\
\hline Lazarus, J.V. 2020 [25] & + & - Trust in the government \\
\hline Wang, J. 2020 [28] & + & $\begin{array}{l}\text { - Perceiving a high risk of infection } \\
\text { - Being vaccinated against influenza in the } \\
\text { previous season }\end{array}$ \\
\hline Lin, C. 2021 [68] & - & $\begin{array}{l}\text { - Belief that vaccines are unnecessary } \\
\text { - Inadequate information } \\
\text { - General anti-vaccine stand } \\
\text { - Willingness to pay }\end{array}$ \\
\hline Murphy, J. 2021 [29] & - & $\begin{array}{l}\text { - Irish sample: more likely to have voted for } \\
\text { the political party Sinn Féin or an } \\
\text { independent politician in the previous } \\
\text { general election }\end{array}$ \\
\hline & + & $\begin{array}{l}\text { - Got a seasonal flu vaccine } \\
\text { - High level of anxiety }\end{array}$ \\
\hline Akarsu, B. 2020 [30] & - & $\begin{array}{l}\text { - "Afraid of the side effects of the vaccines" } \\
\text { - "Do not think it can be reliable as it will be a } \\
\text { new vaccine" } \\
\text { - "COVID-19 infection is a biological weapon" } \\
\text { - "The vaccine will serve those who produce } \\
\text { this virus" }\end{array}$ \\
\hline Hursh, S.R. 2020 [32] & - & $\begin{array}{l}\text { - Greater conspiracy beliefs and political } \\
\text { conservatism }\end{array}$ \\
\hline Biasio, L.R. 2020 [33] & Attitudes to a COVID-19 vaccine & - Health literacy \\
\hline Kourlaba, G. 2021 [34] & + & $\begin{array}{l}\text { - Those believing that the COVID-19 virus } \\
\text { was not developed in laboratories by humans } \\
\text { - Those believing that coronavirus is far more } \\
\text { contagious and lethal compared to the H1N1 } \\
\text { virus } \\
\text { - Those believing that next waves are coming } \\
\text { - Higher knowledge score regarding } \\
\text { symptoms, transmission routes, and } \\
\text { prevention and control measures against } \\
\text { COVID-19 }\end{array}$ \\
\hline Fisher, K.A. 2020 [35] & - & $\begin{array}{l}\text { - Vaccine-specific concerns } \\
\text { - Need for more information } \\
\text { - General anti-vaccine beliefs } \\
\text { - Lack of trust }\end{array}$ \\
\hline Guidry, J.P.D. 2021 [36] & + & $\begin{array}{l}\text { - Positive subjective norms } \\
\text { - A positive attitude toward vaccines in } \\
\text { general } \\
\text { - Perceived susceptibility to COVID-19 } \\
\text { - High perceived benefits of the vaccines } \\
\text { - Scoring low on barriers to the vaccines } \\
\text { - Scoring high on self-efficacy } \\
\text { - High perceived behavioral control }\end{array}$ \\
\hline
\end{tabular}


Table 4. Cont.

\begin{tabular}{|c|c|c|}
\hline First Author & $\begin{array}{c}\text { Hesitancy }(-) \text { or Acceptance }(+) \text { of } \\
\text { COVID-19 Vaccines }\end{array}$ & $\begin{array}{l}\text { Individual Factors Shaping Public } \\
\text { Attitudes towards COVID-19 Vaccines }\end{array}$ \\
\hline Jung, H. 2020 [70] & + & $\begin{array}{l}\text { - Prosocial concern for vaccination motivates } \\
\text { vaccination in more and less populated } \\
\text { regions }\end{array}$ \\
\hline Popa, G.L. 2020 [37] & - & $\begin{array}{l}\text { - Lack of information } \\
\text { - Fear of adverse reactions } \\
\text { - Fears of toxicity and poor quality related to } \\
\text { vaccine components } \\
\text { - Doubts about the technology used to } \\
\text { produce the vaccines } \\
\text { - Personal reasons to refuse vaccines (which } \\
\text { included religious conviction) } \\
\text { - Lack of trust in the healthcare system }\end{array}$ \\
\hline Detoc, M. 2020 [38] & + & $\begin{array}{l}\text { - Fear about COVID-19 and individual } \\
\text { perceived risk }\end{array}$ \\
\hline Prati, G. 2021 [39] & Attitudes to a COVID-19 vaccine & $\begin{array}{l}\text { - Being worried about the non-natural origin } \\
\text { of the virus and the role of the institutional } \\
\text { trust }\end{array}$ \\
\hline Caserotti, M. 2021 [41] & + & $\begin{array}{l}\text { - Likelihood of getting the infection } \\
\text { - Perceived severity of the disease }\end{array}$ \\
\hline Reiter, P.L. 2020 [43] & + & $\begin{array}{l}\text { - Likelihood of getting the COVID-19 } \\
\text { infection in the future } \\
\text { - Perceived severity of the COVID-19 } \\
\text { infection }\end{array}$ \\
\hline Danchin, M. 2020 [65] & + & - Adequate health literacy \\
\hline Harapan, H. 2020 [45] & + & - Perceived risk of the COVID-19 infection \\
\hline Lin, Y. 2020 [46] & + & $\begin{array}{l}\text { - Perceiving overall health as very good } \\
\text { - Perceiving the benefit of feeling less worried } \\
\text { of contracting coronavirus after getting a } \\
\text { vaccine } \\
\text { - Perceiving the benefit of a COVID-19 } \\
\text { vaccine in reducing the risk of infection and } \\
\text { resultant complications } \\
\text { - If given adequate information and if taken } \\
\text { by many in the general public }\end{array}$ \\
\hline Williams, L. 2020 [47] & Attitudes to a COVID-19 vaccine & $\begin{array}{l}\text { - The perception that COVID-19 will persist } \\
\text { over time } \\
\text { - Perceiving the media to have } \\
\text { overexaggerated the risk } \\
\text { - The "beliefs about consequences" TDF } \\
\text { domain, with themes relating to personal } \\
\text { health, health consequences to others, and } \\
\text { severity of COVID-19 }\end{array}$ \\
\hline Alqudeimat, Y. 2021 [48] & - & $\begin{array}{l}\text { - Likelihood of infection } \\
\text { - Viewed vaccines in general to have } \\
\text { health-related risks }\end{array}$ \\
\hline Sallam, M. 2021 [49] & - & $\begin{array}{l}\text { - Beliefs that COVID-19 vaccines are } \\
\text { intended to inject microchips into recipients } \\
\text { and that the vaccines are related to infertility }\end{array}$ \\
\hline Wang, K.L. 2021 [52] & + & $\begin{array}{l}\text { - Influenza vaccine uptake during the } \\
\text { previous year }\end{array}$ \\
\hline
\end{tabular}


Table 4. Cont.

\begin{tabular}{|c|c|c|}
\hline First Author & $\begin{array}{c}\text { Hesitancy }(-) \text { or Acceptance }(+) \text { of } \\
\text { COVID-19 Vaccines }\end{array}$ & $\begin{array}{l}\text { Individual Factors Shaping Public } \\
\text { Attitudes towards COVID-19 Vaccines }\end{array}$ \\
\hline Largent, E.A. 2020 [71] & - & $\begin{array}{l}\text { - Republicans and Independents were less } \\
\text { likely to get vaccinated than Democrats }\end{array}$ \\
\hline Ward, J.K. 2020 [54] & Attitudes to a COVID-19 vaccine & $\begin{array}{l}\text { - Political partisanship and engagement with } \\
\text { the political system }\end{array}$ \\
\hline Romer, D. 2020 [55] & - & $\begin{array}{l}\text { - Belief in three COVID-19-related conspiracy } \\
\text { theories }\end{array}$ \\
\hline Sherman, S.M. 2020 [56] & + & $\begin{array}{l}\text { - Having been vaccinated for influenza the } \\
\text { previous winter } \\
\text { - Perceiving a great risk of COVID-19 } \\
\text { - Positive general COVID-19 vaccination } \\
\text { beliefs and attitudes } \\
\text { - Weak beliefs that the vaccination would } \\
\text { cause side effects } \\
\text { - Greater perceived information sufficiency to } \\
\text { make an informed decision about COVID-19 } \\
\text { vaccination } \\
\text { - Lower endorsement of the notion that only } \\
\text { people who are at risk of serious illness } \\
\text { should be vaccinated for COVID-19 }\end{array}$ \\
\hline McCaffery, K.J. 2020 [57] & - & $\begin{array}{l}\text { - Beliefs and misinformation about } \\
\text { COVID-19/vaccines } \\
\text { - Inadequate health literacy }\end{array}$ \\
\hline Pogue, K. 2020 [58] & + & $\begin{array}{l}\text { - Respondents who routinely got vaccines } \\
\text { were more likely to be receptive to receiving } \\
\text { a COVID-19 vaccine } \\
\text { - The greater the perceived impact of } \\
\text { COVID-19 in America, the more receptive the } \\
\text { respondent was to receive a potential } \\
\text { COVID-19 vaccine }\end{array}$ \\
\hline Taylor, S. 2020 [59] & - & $\begin{array}{l}\text { - Mistrust of vaccine benefits } \\
\text { - Worries about unforeseen future negative } \\
\text { effects } \\
\text { - Concerns about commercial profiteering } \\
\text { - Preference for natural immunity }\end{array}$ \\
\hline Reuben, R.C. 2020 [60] & - & $\begin{array}{l}\text { - No confidence in the present intervention } \\
\text { by Chinese doctors }\end{array}$ \\
\hline \multirow[t]{2}{*}{ Corpuz, R. 2020 [61] } & + & $\begin{array}{l}\text { - Those exhibiting a slow life history } \\
\text { orientation were more likely to endorse } \\
\text { mandatory vaccination for COVID-19 }\end{array}$ \\
\hline & - & - Social and political conservatism \\
\hline Bertin, P. 2020 [62] & - & - COVID-19 conspiracy beliefs \\
\hline Ling, R. 2020 [66] & - & $\begin{array}{l}\text { - Confirmation bias, consumption of only } \\
\text { those news that confirm the pre-existing } \\
\text { attitudes and beliefs }\end{array}$ \\
\hline
\end{tabular}

Our review showed that the individuals who believe that coronavirus is contagious and lethal, have good knowledge and score high on health literacy, are stressed, worried, and anxious about getting infected, trust the healthcare system, the government, and the companies producing the vaccines, and have positive experience with previous vaccines were more likely to accept COVID-19 vaccines than others. On the other hand, the individuals with greater conspiracy beliefs and political conservatism, and those with personal 
reasons to refuse a vaccine, including religious conviction, were hesitant and reported negative attitudes towards COVID-19 vaccines.

\subsection{Social and Organizational Factors Shaping Public Attitudes towards COVID-19 Vaccines}

Table 5 represents the articles that discussed the role of family, friends, healthcare providers, and employers in shaping public attitudes. In addition, it depicts the articles discussing the role of traditional (classic) and social media. Social networks and organizational factors that affect the attitude toward COVID-19 vaccines were reported in 10 studies. Social service and healthcare providers and the physician's recommendation of vaccination $[28,43,44,69]$ were the most prevalent ones. The individuals who were advised by a physician or any other healthcare provider to take a vaccine were more likely to have a positive attitude towards it than those who did not get any advice. The employer's recommendation might also influence an individual's attitude positively [25].

Table 5. Social and organizational factors associated with hesitancy or acceptance of COVID-19 vaccines.

\begin{tabular}{|c|c|c|}
\hline First Author & $\begin{array}{c}\text { Hesitancy }(-) \text { or Acceptance }(+) \text { of } \\
\text { COVID-19 Vaccines }\end{array}$ & $\begin{array}{c}\text { Social Networks and Organizational Factors } \\
\text { (Family, Friends, HC Providers, Employers) } \\
\text { and Media Shaping Public Attitudes towards } \\
\text { COVID-19 Vaccines }\end{array}$ \\
\hline Chen, T.E. 2021 [23] & + & $\begin{array}{l}\text { - Type of messages received and message frames } \\
\text { - Outcome uncertainty } \\
\text { - Number format } \\
\text { - Numeracy skills }\end{array}$ \\
\hline Lazarus, J.V. 2020 [25] & + & $\begin{array}{l}\text { - Accepting their employer's recommendation to } \\
\text { do so }\end{array}$ \\
\hline Wang, J. 2020 [28] & + & - Valuing their doctor's recommendations \\
\hline Seale, H. 2021 [31] & $+/-$ & $\begin{array}{l}\text { - Decision to get vaccinated would be supported } \\
\text { by family and friends }\end{array}$ \\
\hline Popa, G.L. 2020 [37] & - & $\begin{array}{l}\text { - Disinformation (through classic media, social } \\
\text { media, and the Internet) }\end{array}$ \\
\hline Bogart, L.M. 2021 [69] & + & - Social service and healthcare providers \\
\hline Alley, S.J. 2021 [42] & - & - Infrequent users of traditional media \\
\hline Puri, N. 2020 [64] & - & - Anti-vaccination messages on social platforms \\
\hline Reiter, P.L. 2020 [43] & + & $\begin{array}{l}\text { - Healthcare providers recommending } \\
\text { vaccination }\end{array}$ \\
\hline Feleszko, W. 2021 [44] & + & $\begin{array}{l}\text { - Recommended by a family doctor } \\
\text { - Someone of family members/friends was } \\
\text { vaccinated } \\
\text { - Need a vaccination certificate to enter some } \\
\text { countries }\end{array}$ \\
\hline
\end{tabular}

The influence of traditional media [42], type of messages received, and disinformation through the Internet and social media $[23,37,64]$ were among the reported factors. The reviewed articles showed that misleading information shared on social media platforms would make individuals hesitant to take the vaccine. In addition, $78 \%$ of the participants in one study stated that their decision to get a vaccine was supported by their family and friends [31], especially when someone of their family members or friends was vaccinated [44]. 


\subsection{Characteristics of COVID-19 Vaccines and Public Concern}

Table 6 summarizes the literature that shows how some vaccine characteristics affect public attitudes. Efficacy $[28,40,43,45,46,63,65,68,73]$, safety $[25,28,35,47,63,65,68,73]$, cost $[46,63,68,72]$, and adverse effects or toxicity of the vaccine $[37,40,46,47,63,68,72,73]$ were among the vaccine characteristics that were reported to shape public concerns about COVID-19 vaccines. The reviewed articles showed that the individuals who had a negative perception towards vaccine efficacy, safety, and side effects would report unwillingness and hesitancy towards taking a vaccine.

Table 6. Characteristics of COVID-19 vaccines associated with hesitancy or acceptance of the vaccines.

\begin{tabular}{|c|c|c|}
\hline First Author & $\begin{array}{c}\text { Hesitancy }(-) \text { or Acceptance }(+) \text { of } \\
\text { COVID-19 Vaccines }\end{array}$ & $\begin{array}{l}\text { Characteristics of COVID-19 Vaccines Shaping } \\
\text { Public Attitudes towards COVID-19 Vaccines }\end{array}$ \\
\hline Chen, M.2020 [24] & + & - If the vaccine is domestic, not imported \\
\hline Lazarus, J.V. 2020 [25] & + & $\begin{array}{l}\text { - Vaccine proved safe and effective by the } \\
\text { government }\end{array}$ \\
\hline Coustasse, A. 2020 [63] & $+1-$ & $\begin{array}{l}\text { - Effectiveness estimate of the vaccine } \\
\text { - Safety based on newness and adverse effects } \\
\text { - Lack of testing } \\
\text { - Vaccination timeframe } \\
\text { - Who will have access to it } \\
\text { - Cost to consumers } \\
\text { - How states and the federal government will } \\
\text { determine vaccination methods } \\
\text { - Getting COVID-19 from the shot } \\
\text { - Fear of side effects from an untested vaccine }\end{array}$ \\
\hline Robles, A.S. 2020 [73] & $+1-$ & $\begin{array}{l}\text { - Perception of efficacy, safety, and adverse } \\
\text { effects of the vaccines } \\
\text { - Source of information } \\
\text { - Conspiracy theories } \\
\text { - Reactance and outrage regarding new } \\
\text { information }\end{array}$ \\
\hline Wang, J. 2020 [28] & $+1-$ & $\begin{array}{l}\text { - Efficacy of COVID-19 vaccination } \\
\text { - Concerns about vaccine safety }\end{array}$ \\
\hline Lin, C. 2021 [68] & $+/-$ & $\begin{array}{l}\text { - Newness of COVID-19 vaccines } \\
\text { - Inadequate information } \\
\text { - Unknown/short duration of immunity } \\
\text { - Cost } \\
\text { - Country of vaccine origin } \\
\text { - Fear of side effects, safety, and effectiveness }\end{array}$ \\
\hline Fisher, K.A. 2020 [35] & - & $\begin{array}{l}\text { - Vaccine-specific concerns } \\
\text { - Need for more information }\end{array}$ \\
\hline Popa, G.L.P. 2020 [37] & - & $\begin{array}{l}\text { - Fear of adverse reactions } \\
\text { - Fear of toxicity and poor quality related to } \\
\text { vaccine components } \\
\text { - Doubts about the technology used to produce } \\
\text { the vaccines } \\
\text { - Price }\end{array}$ \\
\hline Marco-Franco, J.E. 2021 [40] & - & $\begin{array}{l}\text { - Worries about the side effects, safety and } \\
\text { effectiveness of the vaccines }\end{array}$ \\
\hline Reiter, P.L. 2020 [43] & + & - Effectiveness of a COVID-19 vaccine \\
\hline Danchin, M. 2020 [65] & + & - Vaccine safety and effectiveness \\
\hline Harapan, H. 2020 [45] & + & - The baseline effectiveness of the vaccines \\
\hline
\end{tabular}


Table 6. Cont.

\begin{tabular}{|c|c|c|}
\hline First Author & $\begin{array}{l}\text { Hesitancy }(-) \text { or Acceptance }(+) \text { of } \\
\text { COVID-19 Vaccines }\end{array}$ & $\begin{array}{l}\text { Characteristics of COVID-19 Vaccines Shaping } \\
\text { Public Attitudes towards COVID-19 Vaccines }\end{array}$ \\
\hline Lin, Y. 2020 [46] & $+1-$ & $\begin{array}{l}\text { - Concerns about faulty/fake vaccines } \\
\text { - Affordability and high price } \\
\text { - Safety and efficacy } \\
\text { - Confidence and preference of domestically } \\
\text { made vaccines }\end{array}$ \\
\hline Williams, L. 2020 [47] & $+/-$ & - Personal concerns regarding vaccine safety \\
\hline Yin, F. 2021 [72] & + & $\begin{array}{l}\text { - The majority thought the price was low } \\
\text { - Positive views on side effects } \\
\text { - Information about inactivated vaccines } \\
\text { (inactivated vaccines are more accepted) }\end{array}$ \\
\hline Wang, K.L. 2021 [52] & - & $\begin{array}{l}\text { - Doubts of effectiveness } \\
\text { - Thought of the vaccines as unnecessary } \\
\text { - More accepted in the first wave compared to the } \\
\text { third wave }\end{array}$ \\
\hline Dube, E. 2020 [67] & - & $\begin{array}{l}\text { - Vaccine development is being pushed } \\
\text { - COVID-19 vaccine antigen-carrying platforms } \\
\text { have never been used } \\
\text { - The production of new COVID-19 vaccines will } \\
\text { not meet demand } \\
\text { - Conspiracy theories } \\
\text { - More than one type of COVID-19 vaccines is } \\
\text { likely to be used within a country. Thus, the } \\
\text { safety and efficacy profiles may vary }\end{array}$ \\
\hline
\end{tabular}

Furthermore, immunity duration [68], timeframe of vaccination [63], fake or lowquality vaccines [46], country of vaccine origin $[24,68]$ and information about inactivated vaccines [72] were other public concerns in the selected studies. The individuals who believed that immunity boosted by a vaccine would be for a short period, vaccine development was expedited, and the production process was pushed, and that the vaccines are most probably fake would report negative attitude towards COVID-19 vaccines.

\section{Discussion}

This scoping review of 50 articles systematically maps evidence on the influencing factors that may lead to COVID-19 vaccine hesitancy worldwide. Vaccine hesitancy and antivaccination movements represent an old phenomenon that threatens global health [74-77]. With the current situation of the COVID-19 pandemic, this can be a stumbling block in the global efforts to control the disease and its devastating consequences.

Vaccination is considered a vital element for public health; it is the most effective intervention for the primary prevention of communicable diseases. To enhance acceptance and uptake of vaccines, it is crucial to gain insight into the common factors that influence an individual's decision-making process to help inform policymakers to develop effective strategies. This scoping review updates the latest information on the determinants that impact COVID-19 vaccine uptake. The review included studies that reported global data which offer an insight on how public attitudes towards COVID-19 vaccination varies around the world. It also demonstrated that these attitudes are influenced by a wide range of factors on multiple levels of the socio-ecological model. Sizeable evidence showed that sociodemographic factors such as age, gender, and income status, individual factors such as personal beliefs and risk perception, and social and organizational factors such as the role of significant others are among the most related determinants. In addition, certain characteristics of the COVID-19 vaccines themselves like efficacy, safety, and side effects influence public attitudes (Table S1). 
The individuals' attitudes towards the COVID-19 vaccination varied among the studies, with acceptance ranging from $29.4 \%$ to $86 \%$. This discrepancy could be attributed to variations in the study population. Sallam et al. [49] reported a very low rate of acceptance among people in three Arab countries, Jordan, Kuwait, and Saudi Arabia (29.4\%), as compared to a relatively higher acceptance of $53.1 \%$ reported in one study in Kuwait [48]. In contrast, as high as $86 \%$ of people in UK (mainly elderly and middle-aged at-risk adults) expressed their willingness to receive a vaccine [47]. This is in line with the findings of a recent systematic review [77] that showed a global variation in the rate of vaccine acceptance, with the Middle East being among the regions having the lowest rates. The review related this finding to the widespread embrace of conspiratorial beliefs in the region, which subsequently resulted in negative attitudes towards vaccination. This negative attitude in the Arab region is alarming.

In this review, 28 studies depicted the sociodemographic factors associated with public attitudes toward the COVID-19 vaccination. Coherent to findings from the literature [78], the most common factors found to influence vaccine acceptance at the microlevel were age, educational level, gender, race, and income status [23-36,44-53,63,65,68,71-73]. More willingness to receive a vaccine was reported in the older age group [23,25,27,31,34,35,38,53,65], while resistance, hesitancy, and lack of intention to be vaccinated emerged in the younger age group $[29,35,51]$. This could be attributed to differences in age distribution between countries, literacy level, and the fact that older adults are at a higher risk of morbidity and mortality than young adults.

Assessment of the role of gender in COVID-19 vaccine hesitancy revealed that men are more willing to accept the vaccine than women [28-30,38,48,49], and this held true across cultures (Arab countries [48,49], China [28], Ireland, and the UK [29]). Only one cross-sectional study in the USA reported lower acceptance among men [32]. Women were reported to have adopted more negative views about vaccination [30] while men showed a lower belief in rumors and conspiracy theories surrounding COVID-19 and higher risk perception for the disease [49,77]. However, this finding should be interpreted with caution in light of sex distribution, as sampling bias cannot be ruled out.

Similar to previous findings, the current review found variations in vaccine acceptance and uptake across different race and ethnic minorities. Blacks, Hispanics, Chinese, Asian, non-Irish, mixed, or other ethnicities were more hesitant and more likely to reject the vaccines. The literature attributed this attitude to religious and cultural beliefs, norms, and concerns $[37,79]$. High education level and high-income status were associated with positive attitudes toward vaccination, owing to minimal barriers related to knowledge, health literacy, and cost concerns [23-25,27,30,35,36,42,47,49-51,63,65].

In addition, other factors were included, such as health condition, people with chronic diseases, occupation status, marital status, place of residence, women being pregnant or having children, and having health insurance or not. More willingness and acceptance were reported among married individuals $[7,28,52]$, those who reside in rural or suburban areas $[29,68]$, being employed $[27,46,50,53,73]$, especially in professional and managerial occupations [50,53], being at risk or belonging to a vulnerable group [34,45,65], and having insurance $[30,31,36,51,68]$. Knowing these factors can provide guidance for organizations and professionals on people and settings that need to be targeted to enhance vaccine acceptance and improve vaccine uptake rates [79].

More publications ( 36 studies) reported several individual factors influencing public attitudes towards COVID-19 vaccines. The most cited factors were beliefs [30,32,34,35,49,55-57,62,66,68,70], knowledge, and health literacy $[23,33-35,39,57,65,68]$. Other factors such as perceived susceptibility, threats and benefits, social, religious, and political views, previous exposure to flu vaccines, and lack of trust in the governments and companies producing the vaccines were also reported. This is consistent with findings from other reviews related to COVID-19 vaccines and other vaccines $[77,78,80]$.

Knowledge about COVID-19 vaccines is limited as illustrated in numerous studies [23, $33-35,39,57,65,68]$. Unfavorable attitudes toward vaccination was related to misbeliefs, conspir- 
acy beliefs, and antivaccine beliefs [35,47,49,56,57,62,66,68], inadequate knowledge and health literacy $[33,57,65]$, lower perceived risk, threat, severity, and susceptibility $[24,28,38,41,43,45-48,56]$, political conservatism, partisanship and engagement $[29,32,54,61,66]$, and religious conviction [37]. Nevertheless, the factors associated with more vaccination acceptance included positive subjective norms and attitudes towards vaccination in general and COVID-19 vaccination in particular $[36,56,59,66]$, high perceived benefits [46], self-efficacy [36], institutional and government trust $[25,35,37,39,59]$, previous exposure to flu or other vaccines $[28,30,52,56,58]$, and prosocial concerns $[61,70]$. Enhancing these factors may improve the vaccination uptake rate.

Several studies examined the role of the social network and organizational factors $[24,25,28,31,37,42-44,64,69]$. Healthcare professionals appeared to be a trusted source of information. Their recommendations $[28,43,44,69]$ in addition to support of family and friends [31,44] play an important role in shaping perceptions and behaviors towards vaccination. Significant others were reported in the literature to influence one's attitude and behavior. Information, acknowledgement, and recommendations from family members, friends, employers, and community members were associated with favorable attitudes and a higher uptake of vaccines $[25,78,80]$.

On the other hand, the misinformation encountered, particularly on social media, the type and frame of massages received may influence the attitude to vaccination and intention $[24,37,42,64]$. Propagation of myths and conspiracy theories around vaccines and promotion of the antivaccine sentiment, combined with exposure to persuasive tactics, can convince the person that the vaccine is harmful, as indicated by Sarah Ashfield et al. [81]. Accordingly, public health organizations, healthcare professionals, and media platforms can collaborate to guarantee information accuracy, deliver health promotion programs to improve levels of health literacy to enable the target population to make an informed decision. In addition, this psychosocial environmental impact implies that strategies to overcome hesitancy can be framed within models that consider these multifaceted and multileveled factors.

Regarding vaccine characteristics, many publications included in this review focused on efficacy, safety, adverse effects or toxicity of the vaccines, and cost [28,35,40,43,45-47,63,65,68,72,73], which were the most significant characteristics and concerns about COVID-19 vaccines. These were also common factors highlighted in other reviews about COVID-19 vaccines and other vaccines $[77,78,80]$.

Beyond these, the present review also found other factors that further contribute to our understanding of the barriers to vaccination uptake. Immunity duration [68], vaccination timeframe [63], fake or low-quality vaccines [37,46], beliefs about the consequences [47], country of vaccine origin [68], information about inactivated vaccines [72], and doubts about technology used in production $[37,67]$ allow a detailed understanding of how to approach vaccine-hesitant groups to increase acceptance and uptake of the vaccines. These concerns can be addressed via awareness campaigns guided by physicians and other healthcare professionals to foster trust in health authorities, assure the public, and illustrate the role of vaccination in acquiring herd immunity and preventing disease transmission.

As illustrated above, the majority of the studies in this review addressed factors associated with vaccination attitude at the micro-meso level; however, there is a lack of publications that address the factors on the upper level of the socio-ecological model. Determinants on the macro-level of the model, including policy/regulations, broad sociocultural, religious, political, and environmental factors, and the influence they may exert on COVID-19 vaccine uptake are underexplored to date, at least in the results presented in our review. This gap in evidence necessitates further research to comprehensively tackle the issue of vaccine hesitancy. Another important output for this scoping review reflects the gap in clinical evidence concerning the COVID-19 vaccine efficacy, safety, and side effects to date, which showed an influence in shaping public hesitancy and refusal of the vaccines. Clinical research is needed to fill this gap and manage sharing evidence that will alleviate public concerns and enhance vaccine acceptance. 
The included studies reported global data which can be seen as a strength of this review. On the other hand, our review has some limitations. Only the articles in English were included; this may have potentially introduced bias or resulted in missing important literature. We did not include one keyword, "Antivax," in our search strategy; however, when we did, only one new publication was found, therefore, this would unlikely have a noticeable effect on our results. The majority of the included studies are cross-sectional, which limits the ability to infer the causation between the various factors and public attitudes towards the COVID-19 vaccination. Most of these studies used self-administered surveys that may lead to biases. Furthermore, uncontrolled health conditions of the target populations and the various global healthcare systems in the studies included in this review may have had a misleading influence on the results.

Understanding various population needs and the factors shaping public attitudes towards the vaccines would support planning for evidence-based multilevel interventions in order to enhance the vaccine uptake globally. In our findings, we were able to report factors on the individual, social, and organizational levels. Future research should focus on exploring the cultural, economic, and political factors influencing public attitudes towards the COVID-19 vaccination.

\section{Conclusions}

This review highlights the complexity of the topic. Our findings show that attitudes toward COVID-19 vaccines is shaped by factors that are multifaceted and multileveled. A combination of a set of complementary multilevel interventions and engagement of diverse players, recipients, and settings may be helpful to improve the vaccination uptake to win the fight against this pandemic.

Supplementary Materials: The following are available online at https://www.mdpi.com/article/ 10.3390 /vaccines $9060548 / \mathrm{s} 1$, Table S1. Summary table of the factors associated with hesitancy or acceptance of COVID-19 vaccines.

Author Contributions: Conceptualization, G.F.A.-J., M.A.M.S., and A.E.-H.; methodology, G.F.A.-J., M.A.M.S., and M.A.N.; records identification, abstracts, and citations screening, G.F.A.-J. and M.A.M.S.; assessment of the full texts of the relevant records, G.F.A.-J., M.A.M.S., A.E.-H., N.W.Z.A., and M.E.A.E.; writing-original draft preparation, G.F.A.-J., L.A.M.A., N.W.Z.A., and M.A.N.; writing-review and editing, G.F.A.-J., M.A.M.S., L.A.M.A., A.E.-H., and M.E.A.E.; project administration, G.F.A.-J. All authors have read and agreed to the published version of the manuscript.

Funding: This research received no external funding.

Institutional Review Board Statement: Not applicable.

Informed Consent Statement: Not applicable.

Data Availability Statement: The data presented in this study are available in Supplementary materials.

Conflicts of Interest: The authors declare no conflict of interest. 


\section{Appendix A}

Table A1. Preferred Reporting Items for Systematic Reviews and Meta-Analyses extension for Scoping Reviews (PRISMA-ScR) Checklist.

\begin{tabular}{|c|c|c|c|}
\hline Section & Item & Prisma-ScR Checklist ITEM & Reported on Page \# \\
\hline \multicolumn{4}{|c|}{ Title } \\
\hline Title & 1 & Identify the report as a scoping review. & 1 \\
\hline \multicolumn{4}{|c|}{ Abstract } \\
\hline Structured summary & 2 & $\begin{array}{l}\text { Provide a structured summary that includes } \\
\text { (as applicable): background, objectives, } \\
\text { eligibility criteria, sources of evidence, charting } \\
\text { methods, results, and conclusions that relate to } \\
\text { the review questions and objectives. }\end{array}$ & 1 \\
\hline \multicolumn{4}{|c|}{ Introduction } \\
\hline Rationale & 3 & $\begin{array}{l}\text { Describe the rationale for the review in the } \\
\text { context of what is already known. Explain why } \\
\text { the review questions/objectives lend } \\
\text { themselves to a scoping review approach. }\end{array}$ & 2 \\
\hline Objectives & 4 & $\begin{array}{l}\text { Provide an explicit statement of the questions } \\
\text { and objectives being addressed with reference } \\
\text { to their key elements (e.g., population or } \\
\text { participants, concepts, and context) or other } \\
\text { relevant key elements used to conceptualize } \\
\text { the review questions and/or objectives. }\end{array}$ & 3 \\
\hline \multicolumn{4}{|c|}{ Methods } \\
\hline Protocol and registration & 5 & $\begin{array}{l}\text { Indicate whether a review protocol exists; state } \\
\text { if and where it can be accessed (e.g., a Web } \\
\text { address); and if available, provide registration } \\
\text { information, including the registration number. }\end{array}$ & 3 \\
\hline Eligibility criteria & 6 & $\begin{array}{l}\text { Specify characteristics of the sources of } \\
\text { evidence used as eligibility criteria (e.g., years } \\
\text { considered, language, and publication status), } \\
\text { and provide a rationale. }\end{array}$ & 3 \\
\hline Information sources * & 7 & $\begin{array}{l}\text { Describe all information sources in the search } \\
\text { (e.g., databases with dates of coverage and } \\
\text { contact with authors to identify additional } \\
\text { sources), as well as the date the most recent } \\
\text { search was executed. }\end{array}$ & 3 \\
\hline Search & 8 & $\begin{array}{l}\text { Present the full electronic search strategy for at } \\
\text { least } 1 \text { database, including any limits used, } \\
\text { such that it could be repeated. }\end{array}$ & 3 \\
\hline $\begin{array}{c}\text { Selection of sources of } \\
\text { evidence }+\end{array}$ & 9 & $\begin{array}{l}\text { State the process for selecting sources of } \\
\text { evidence (i.e., screening and eligibility) } \\
\text { included in the scoping review. }\end{array}$ & 3 \\
\hline Data charting process $\ddagger$ & 10 & $\begin{array}{l}\text { Describe the methods of charting data from the } \\
\text { included sources of evidence (e.g., calibrated } \\
\text { forms or forms that have been tested by the } \\
\text { team before their use, and whether data } \\
\text { charting was done independently or in } \\
\text { duplicate) and any processes for obtaining and } \\
\text { confirming data from investigators. }\end{array}$ & 4 \\
\hline Data items & 11 & $\begin{array}{l}\text { List and define all variables for which data } \\
\text { were sought and any assumptions and } \\
\text { simplifications made. }\end{array}$ & 4 \\
\hline
\end{tabular}


Table A1. Cont.

\begin{tabular}{|c|c|c|c|}
\hline Section & Item & Prisma-ScR Checklist ITEM & Reported on Page \# \\
\hline $\begin{array}{l}\text { Critical appraisal of } \\
\text { individual sources of } \\
\text { evidence } \S\end{array}$ & 12 & $\begin{array}{l}\text { If done, provide a rationale for conducting a } \\
\text { critical appraisal of included sources of } \\
\text { evidence; describe the methods used and how } \\
\text { this information was used in any data } \\
\text { synthesis (if appropriate). }\end{array}$ & NA \\
\hline Synthesis of results & 13 & $\begin{array}{l}\text { Describe the methods of handling and } \\
\text { summarizing the data that were charted. }\end{array}$ & 4 \\
\hline \multicolumn{4}{|c|}{ Results } \\
\hline $\begin{array}{l}\text { Selection of sources of } \\
\text { evidence }\end{array}$ & 14 & $\begin{array}{l}\text { Give numbers of sources of evidence screened, } \\
\text { assessed for eligibility, and included in the } \\
\text { review, with reasons for exclusions at each } \\
\text { stage, ideally using a flow diagram. }\end{array}$ & 5 \\
\hline $\begin{array}{l}\text { Characteristics of sources } \\
\text { of evidence }\end{array}$ & 15 & $\begin{array}{l}\text { For each source of evidence, present } \\
\text { characteristics for which data were charted and } \\
\text { provide the citations. }\end{array}$ & 6 \\
\hline $\begin{array}{l}\text { Critical appraisal within } \\
\text { sources of evidence }\end{array}$ & 16 & $\begin{array}{l}\text { If done, present data on critical appraisal of } \\
\text { included sources of evidence (see item 12). }\end{array}$ & NA \\
\hline $\begin{array}{l}\text { Results of individual } \\
\text { sources of evidence }\end{array}$ & 17 & $\begin{array}{l}\text { For each included source of evidence, present } \\
\text { the relevant data that were charted that relate } \\
\text { to the review questions and objectives. }\end{array}$ & 8 \\
\hline Synthesis of results & 18 & $\begin{array}{c}\text { Summarize and/or present the charting results } \\
\text { as they relate to the review questions and } \\
\text { objectives. }\end{array}$ & 13 \\
\hline
\end{tabular}

Discussion

Summarize the main results (including an overview of concepts, themes, and types of

evidence available), link to the review questions and objectives, and consider the relevance to key groups.

\begin{tabular}{ccc}
\hline Limitations & 20 & $\begin{array}{c}\text { Discuss the limitations of the scoping review } \\
\text { process. }\end{array}$ \\
\hline Conclusions & $21 \quad \begin{array}{c}\text { Provide a general interpretation of the results } \\
\text { with respect to the review questions and } \\
\text { objectives, as well as potential implications } \\
\text { and/or next steps. }\end{array}$ \\
\hline
\end{tabular}

\section{Funding}

Funding
Describe sources of funding for the included sources of evidence, as well as sources of funding for the scoping review. Describe the role of the funders of the scoping review.

JBI = Joanna Briggs Institute; PRISMA-ScR = Preferred Reporting Items for Systematic Reviews and Meta-Analyses extension for Scoping Reviews. * Where sources of evidence (see second footnote) are compiled from, such as bibliographic databases, social media platforms, and Web sites. + A more inclusive/heterogeneous term used to account for the different types of evidence or data sources (e.g., quantitative and/or qualitative research, expert opinion, and policy documents) that may be eligible in a scoping review as opposed to only studies. This is not to be confused with information sources (see first footnote). $\ddagger$ The frameworks by Arksey and O'Malley (6) and Levac and colleagues $(7)$ and the JBI guidance $(4,5)$ refer to the process of data extraction in a scoping review as data charting. $\S$ The process of systematically examining research evidence to assess its validity, results, and relevance before using it to inform a decision. This term is used for items 12 and 19 instead of "risk of bias" (which is more applicable to systematic reviews of interventions) to include and acknowledge the various sources of evidence that may be used in a scoping review (e.g., quantitative and/or qualitative research, expert opinion, and policy document). 


\section{References}

1. Zhu, H.; Wei, L.; Niu, P. The novel coronavirus outbreak in Wuhan, China. Glob. Health Res. Policy 2020, 5, 1-3. [CrossRef]

2. Worldometer. COVID-19 Coronavirus Pandemic. Available online: https://www.worldometers.info/coronavirus/?utm_ campaign=homeAdvegas1? (accessed on 21 March 2021).

3. Nicola, M.; Alsafi, Z.; Sohrabi, C.; Kerwan, A.; Al-Jabir, A.; Iosifidis, C.; Agha, M.; Agha, R. The socio-economic implications of the coronavirus pandemic (COVID-19): A review. Int. J. Surg. 2020, 78, 185-193. [CrossRef]

4. Phua, J.; Weng, L.; Ling, L.; Egi, M.; Lim, C.-M.; Divatia, J.V.; Shrestha, B.R.; Arabi, Y.M.; Ng, J.; Gomersall, C.D.; et al. Intensive care management of coronavirus disease 2019 (COVID-19): Challenges and recommendations. Lancet Respir. Med. 2020, 8 , 506-517. [CrossRef]

5. World Health Organization. Modes of Transmission of Virus Causing COVID-19: Implications for IPC Precaution Recommendations. Available online: https://www.who.int/news-room/commentaries/detail/modes-of-transmission-of-virus-causingcovid-19-implications-for-ipc-precaution-recommendations (accessed on 21 March 2021).

6. World Health Organization. Coronavirus. Available online: https://www.who.int/health-topics/coronavirus\#tab=tab_1 (accessed on 21 March 2021).

7. Ferguson, N.M.; Laydon, D.; Nedjati-Gilani, G.; Imai, N.; Ainslie, K.; Baguelin, M.; Ghani, A.C. Impact of Non-Pharmaceutical Interventions (Npis) to Reduced Covid-19 Mortality and Healthcare Demand; Imperial College London: London, UK, 2020.

8. Yamey, G.; Schäferhoff, M.; Hatchett, R.; Pate, M.; Zhao, F.; McDade, K.K. Ensuring global access to COVID-19 vaccines. Lancet 2020, 395, 1405-1406. [CrossRef]

9. Centers for Disease Control and Prevention. Vaccines: The Basics. Available online: https://www.cdc.gov/vaccines/vpd/vpdvac-basics.html (accessed on 21 March 2021).

10. Orenstein, W.A.; Ahmed, R. Simply put: Vaccination saves lives. Proc. Natl. Acad. Sci. USA 2017, 114, 4031-4033. [CrossRef]

11. Butter, S.; McGlinchey, E.; Berry, E.; Armour, C. Psychological, social, and situational factors associated with COVID-19 vaccination intentions: A study of UK key workers and non-key workers. Br. J. Health Psychol. 2021, 1-17. [CrossRef]

12. Dror, A.A.; Eisenbach, N.; Taiber, S.; Morozov, N.G.; Mizrachi, M.; Zigron, A.; Srouji, S.; Sela, E. Vaccine hesitancy: The next challenge in the fight against COVID-19. Eur. J. Epidemiol. 2020, 35, 775-779. [CrossRef]

13. Geoghegan, S.; O'Callaghan, K.P.; Offit, P.A. Vaccine Safety: Myths and Misinformation. Front. Microbiol. 2020, 11, 372. [CrossRef]

14. Bendau, A.; Plag, J.; Petzold, M.B.; Ströhle, A. COVID-19 vaccine hesitancy and related fears and anxiety. Int. Immunopharmacol. 2021, 97, 107724. [CrossRef]

15. Wilson, K.; Nguyen, T.; Henningsen, K.H.; Brehaut, J.C.; Hoe, E. Acceptance of a pandemic influenza vaccine: A systematic review of surveys of the general public. Infect. Drug Resist. 2011, 4, 197-207. [CrossRef]

16. Dubé, E.; Laberge, C.; Guay, M.; Bramadat, P.; Roy, R.; Bettinger, J.A. Vaccine hesitancy. Hum. Vaccines Immunother. 2013, 9 , 1763-1773. [CrossRef]

17. Paul, E.; Steptoe, A.; Fancourt, D. Attitudes towards vaccines and intention to vaccinate against COVID-19: Implications for public health communications. Lancet Reg. Health Eur. 2021, 1, 100012. [CrossRef]

18. Johnson, N.F.; Velásquez, N.; Restrepo, N.J.; Leahy, R.; Gabriel, N.; El Oud, S.; Zheng, M.; Manrique, P.; Wuchty, S.; Lupu, Y. The online competition between pro- and anti-vaccination views. Nature 2020, 582, 230-233. [CrossRef]

19. Bish, A.; Michie, S. Demographic and attitudinal determinants of protective behaviours during a pandemic: A review. Br. J. Health Psychol. 2010, 15, 797-824. [CrossRef]

20. Habersaat, K.B.; Betsch, C.; Danchin, M.; Sunstein, C.R.; Böhm, R.; Falk, A.; Brewer, N.T.; Omer, S.B.; Scherzer, M.; Sah, S.; et al. Ten considerations for effectively managing the COVID-19 transition. Nat. Hum. Behav. 2020, 4, 677-687. [CrossRef]

21. Tricco, A.C.; Lillie, E.; Zarin, W.; O’Brien, K.K.; Colquhoun, H.; Levac, D.; Moher, D.; Peters, M.D.; Horsley, T.; Weeks, L.; et al. PRISMA extension for scoping reviews (PRISMA-ScR): Checklist and explanation. Ann. Intern. Med. 2018, 169, 467-473. [CrossRef]

22. Dahlgren, G.; Whitehead, M. Policies and Strategies to Promote Social Equity in Health. Background Document to WHO-Strategy Paper for Europe; Institute for Futures Studies: Stockholm, Sweden, 1991.

23. Chen, T.; Dai, M.; Xia, S.; Zhou, Y. Do Messages Matter? Investigating the Combined Effects of Framing, Outcome Uncertainty, and Number Format on COVID-19 Vaccination Attitudes and Intention. Health Commun. 2021, 1-8. [CrossRef]

24. Chen, M.; Li, Y.; Chen, J.; Wen, Z.; Feng, F.; Zou, H.; Fu, C.; Chen, L.; Shu, Y.; Sun, C. An online survey of the attitude and willingness of Chinese adults to receive COVID-19 vaccination. Hum. Vaccines Immunother. 2021, 1-10. [CrossRef]

25. Lazarus, J.V.; Ratzan, S.C.; Palayew, A.; Gostin, L.O.; Larson, H.J.; Rabin, K.; Kimball, S.; El-Mohandes, A. A global survey of potential acceptance of a COVID-19 vaccine. Nat. Med. 2021, 27, 225-228. [CrossRef]

26. Bell, S.; Clarke, R.; Mounier-Jack, S.; Walker, J.L.; Paterson, P. Parents' and guardians' views on the acceptability of a future COVID-19 vaccine: A multi-methods study in England. Vaccine 2020, 38, 7789-7798. [CrossRef]

27. Al-Mohaithef, M.; Padhi, B.K. Determinants of COVID-19 Vaccine Acceptance in Saudi Arabia: A Web-Based National Survey. J. Multidiscip. Healthc. 2020, 13, 1657-1663. [CrossRef]

28. Wang, J.; Jing, R.; Lai, X.; Zhang, H.; Lyu, Y.; Knoll, M.D.; Fang, H. Acceptance of COVID-19 Vaccination during the COVID-19 Pandemic in China. Vaccines 2020, 8, 482. [CrossRef] 
29. Murphy, J.; Vallières, F.; Bentall, R.P.; Shevlin, M.; McBride, O.; Hartman, T.K.; McKay, R.; Bennett, K.; Mason, L.; Gibson-Miller, J.; et al. Psychological characteristics associated with COVID-19 vaccine hesitancy and resistance in Ireland and the United Kingdom. Nat. Commun. 2021, 12, 1-15. [CrossRef] [PubMed]

30. Akarsu, B.; Özdemir, D.C.; Baser, D.A.; Aksoy, H.; Fidanc1, I.; Cankurtaran, M. While studies on COVID-19 vaccine is ongoing, the public's thoughts and attitudes to the future COVID-19 vaccine. Int. J. Clin. Pract. 2021, 75, e13891. [CrossRef]

31. Seale, H.; Heywood, A.E.; Leask, J.; Sheel, M.; Durrheim, D.N.; Bolsewicz, K.; Kaur, R. Examining Australian public perceptions and behaviors towards a future COVID-19 vaccine. BMC Infect. Dis. 2021, 21, 1-9. [CrossRef] [PubMed]

32. Hursh, S.R.; Strickland, J.C.; Schwartz, L.P.; Reed, D.D. Quantifying the Impact of Public Perceptions on Vaccine Acceptance Using Behavioral Economics. Front. Public Health 2020, 8, 608852. [CrossRef] [PubMed]

33. Biasio, L.R.; Bonaccorsi, G.; Lorini, C.; Pecorelli, S. Assessing COVID-19 vaccine literacy: A preliminary online survey. Hum. Vaccines Immunother. 2021, 17, 1304-1312. [CrossRef]

34. Kourlaba, G.; Kourkouni, E.; Maistreli, S.; Tsopela, C.-G.; Molocha, N.-M.; Triantafyllou, C.; Koniordou, M.; Kopsidas, I.; Chorianopoulou, E.; Maroudi-Manta, S.; et al. Willingness of Greek general population to get a COVID-19 vaccine. Glob. Health Res. Policy 2021, 6, 1-10. [CrossRef]

35. Fisher, K.A.; Bloomstone, S.J.; Walder, J.; Crawford, S.; Fouayzi, H.; Mazor, K.M. Attitudes toward a potential SARS-CoV-2 vaccine: A survey of US adults. Ann. Intern. Med. 2020, 173, 964-973. [CrossRef]

36. Guidry, J.P.; Laestadius, L.I.; Vraga, E.K.; Miller, C.A.; Perrin, P.B.; Burton, C.W.; Ryan, M.; Fuemmeler, B.F.; Carlyle, K.E. Willingness to get the COVID-19 vaccine with and without emergency use authorization. Am. J. Infect. Control 2021, 49, 137-142. [CrossRef]

37. Popa, G.L.; Muntean, A.-A.; Muntean, M.-M.; Popa, M.I. Knowledge and Attitudes on Vaccination in Southern Romanians: A Cross-Sectional Questionnaire. Vaccines 2020, 8, 774. [CrossRef]

38. Detoc, M.; Bruel, S.; Frappe, P.; Tardy, B.; Botelho-Nevers, E.; Gagneux-Brunon, A. Intention to participate in a COVID-19 vaccine clinical trial and to get vaccinated against COVID-19 in France during the pandemic. Vaccine 2020, 38, 7002-7006. [CrossRef]

39. Prati, G. Intention to receive a vaccine against SARS-CoV-2 in Italy and its association with trust, worry and beliefs about the origin of the virus. Health Educ. Res. 2020, 35, 505-511. [CrossRef]

40. Marco-Franco, J.E.; Pita-Barros, P.; Vivas-Orts, D.; González-De-Julián, S.; Vivas-Consuelo, D. COVID-19, Fake News, and Vaccines: Should Regulation Be Implemented? Int. J. Environ. Res. Public Health 2021, 18, 744. [CrossRef]

41. Caserotti, M.; Girardi, P.; Rubaltelli, E.; Tasso, A.; Lotto, L.; Gavaruzzi, T. Associations of COVID-19 risk perception with vaccine hesitancy over time for Italian residents. Soc. Sci. Med. 2021, 272, 113688. [CrossRef]

42. Alley, S.J.; Stanton, R.; Browne, M.; To, Q.G.; Khalesi, S.; Williams, S.L.; Thwaite, T.; Fenning, A.; Vandelanotte, C. As the Pandemic Progresses, How Does Willingness to Vaccinate against COVID-19 Evolve? Int. J. Environ. Res. Public Health 2021, $18,797$. [CrossRef]

43. Reiter, P.L.; Pennell, M.L.; Katz, M.L. Acceptability of a COVID-19 vaccine among adults in the United States: How many people would get vaccinated? Vaccine 2020, 38, 6500-6507. [CrossRef]

44. Feleszko, W.; Lewulis, P.; Czarnecki, A.; Waszkiewicz, P. Flattening the Curve of COVID-19 Vaccine Rejection-An International Overview. Vaccines 2021, 9, 44. [CrossRef]

45. Harapan, H.; Wagner, A.L.; Yufika, A.; Winardi, W.; Anwar, S.; Gan, A.K.; Setiawan, A.M.; Rajamoorthy, Y.; Sofyan, H.; Mudatsir, M. Acceptance of a COVID-19 Vaccine in Southeast Asia: A Cross-Sectional Study in Indonesia. Front. Public Health 2020, 8, 381. [CrossRef]

46. Lin, Y.; Hu, Z.; Zhao, Q.; Alias, H.; Danaee, M.; Wong, L.P. Understanding COVID-19 vaccine demand and hesitancy: A nationwide online survey in China. PLoS Negl. Trop. Dis. 2020, 14, e0008961. [CrossRef]

47. Williams, L.; Gallant, A.J.; Rasmussen, S.; Nicholls, L.A.B.; Cogan, N.; Deakin, K.; Young, D.; Flowers, P. Towards intervention development to increase the uptake of COVID-19 vaccination among those at high risk: Outlining evidence-based and theoretically informed future intervention content. Br. J. Health Psychol. 2020, 25, 1039-1054. [CrossRef] [PubMed]

48. Alqudeimat, Y.; Alenezi, D.; AlHajri, B.; Alfouzan, H.; Almokhaizeem, Z.; Altamimi, S.; Almansouri, W.; Alzalzalah, S.; Ziyab, A. Acceptance of a COVID-19 Vaccine and its Related Determinants among the General Adult Population in Kuwait. Med. Princ. Pract. 2021, 10, 2052-2061. [CrossRef]

49. Sallam, M.; Dababseh, D.; Eid, H.; Al-Mahzoum, K.; Al-Haidar, A.; Taim, D.; Yaseen, A.; Ababneh, N.A.; Bakri, F.G.; Mahafzah, A. High Rates of COVID-19 Vaccine Hesitancy and Its Association with Conspiracy Beliefs: A Study in Jordan and Kuwait among Other Arab Countries. Vaccines 2021, 9, 42. [CrossRef] [PubMed]

50. Wong, L.P.; Alias, H.; Wong, P.-F.; Lee, H.Y.; Abubakar, S. The use of the health belief model to assess predictors of intent to receive the COVID-19 vaccine and willingness to pay. Hum. Vaccines Immunother. 2020, 16, 1232-1238. [CrossRef] [PubMed]

51. Nguyen, K.H.; Srivastav, A.; Razzaghi, H.; Williams, W.; Lindley, M.C.; Jorgensen, C.; Abad, N.; Singleton, J.A. COVID-19 Vaccination Intent, Perceptions, and Reasons for Not Vaccinating Among Groups Prioritized for Early Vaccination-United States, September and December 2020. MMWR Morb. Mortal. Wkly. Rep. 2021, 70, 217-222. [CrossRef] [PubMed]

52. Wang, K.; Wong, E.L.Y.; Ho, K.-F.; Cheung, A.W.L.; Yau, P.S.Y.; Dong, D.; Wong, S.Y.S.; Yeoh, E.-K. Change of willingness to accept COVID-19 vaccine and reasons of vaccine hesitancy of working people at different waves of local epidemic in Hong Kong, China: Repeated cross-sectional surveys. Vaccines 2021, 9, 62. [CrossRef] 
53. La Vecchia, C.; Negri, E.; Alicandro, G.; Scarpino, V. Attitudes towards influenza vaccine and a potential COVID-19 vaccine in Italy and differences across occupational groups, September 2020. Med. Del. Lavoro 2020, 111, 445-448.

54. Ward, J.K.; Alleaume, C.; Peretti-Watel, P.; Seror, V.; Cortaredona, S.; Launay, O.; Raude, J.; Verger, P.; Beck, F.; Legleye, S.; et al. The French public's attitudes to a future COVID-19 vaccine: The politicization of a public health issue. Soc. Sci. Med. 2020, 265, 113414. [CrossRef]

55. Romer, D.; Jamieson, K.H. Conspiracy theories as barriers to controlling the spread of COVID-19 in the U.S. Soc. Sci. Med. 2020, 263, 113356. [CrossRef]

56. Sherman, S.M.; Smith, L.E.; Sim, J.; Amlôt, R.; Cutts, M.; Dasch, H.; Rubin, G.J.; Sevdalis, N. COVID-19 vaccination intention in the UK: Results from the COVID-19 vaccination acceptability study (CoVAccS), a nationally representative cross-sectional survey. Hum. Vaccines Immunother. 2021, 17, 1612-1621. [CrossRef]

57. McCaffery, K.J.; Dodd, R.H.; Cvejic, E.; Ayrek, J.; Isautier, J.M.; Copp, T.; Bonner, C.; Pickles, K.; Nickel, B.; Dakin, T.; et al. Health literacy and disparities in COVID-19-related knowledge, attitudes, beliefs and behaviours in Australia. Public Health Res. Pr. 2020, 30. [CrossRef]

58. Pogue, K.; Jensen, J.L.; Stancil, C.K.; Ferguson, D.G.; Hughes, S.J.; Mello, E.J.; Burgess, R.; Berges, B.K.; Quaye, A.; Poole, B.D. Influences on Attitudes Regarding Potential COVID-19 Vaccination in the United States. Vaccines 2020, 8, 582. [CrossRef] [PubMed]

59. Taylor, S.; Landry, C.A.; Paluszek, M.M.; Groenewoud, R.; Rachor, G.S.; Asmundson, G.J.G. A Proactive Approach for Managing COVID-19: The Importance of Understanding the Motivational Roots of Vaccination Hesitancy for SARS-CoV2. Front. Psychol. 2020, 11, 575950. [CrossRef]

60. Reuben, R.C.; Danladi, M.M.A.; Saleh, D.A.; Ejembi, P.E. Knowledge, Attitudes and Practices towards COVID-19: An Epidemiological Survey in North-Central Nigeria. J. Community Health 2021, 46, 457-470. [CrossRef]

61. Corpuz, R.; D’Alessandro, S.; Adeyemo, J.; Jankowski, N.; Kandalaft, K. Life History Orientation Predicts COVID-19 Precautions and Projected Behaviors. Front. Psychol. 2020, 11, 1857. [CrossRef] [PubMed]

62. Bertin, P.; Nera, K.; Delouvée, S. Conspiracy Beliefs, Rejection of Vaccination, and Support for hydroxychloroquine: A Conceptual Replication-Extension in the COVID-19 Pandemic Context. Front. Psychol. 2020, 11, 11. [CrossRef]

63. Coustasse, A.; Kimble, C.; Maxik, K. COVID-19 and Vaccine Hesitancy. J. Ambul. Care Manag. 2021, 44, 71-75. [CrossRef] [PubMed]

64. Puri, N.; Coomes, E.A.; Haghbayan, H.; Gunaratne, K. Social media and vaccine hesitancy: New updates for the era of COVID-19 and globalized infectious diseases. Hum. Vaccines Immunother. 2020, 16, 2586-2593. [CrossRef]

65. Danchin, M.; Biezen, R.; Manski-Nankervis, J.-A.; Kaufman, J.; Leask, J. Preparing the public for COVID-19 vaccines: How can general practitioners build vaccine confidence and optimise uptake for themselves and their patients? Aust. J. Gen. Pract. 2020, 49, 625-629. [CrossRef]

66. Ling, R. Confirmation Bias in the Era of Mobile News Consumption: The Social and Psychological Dimensions. Digit. J. 2020, 8, 596-604. [CrossRef]

67. Dubé, E.; MacDonald, N.E. How can a global pandemic affect vaccine hesitancy? Expert Rev. Vaccines 2020, 19, 899-901. [CrossRef] [PubMed]

68. Lin, C.; Tu, P.; Beitsch, L.M. Confidence and Receptivity for COVID-19 Vaccines: A Rapid Systematic Review. Vaccines 2021, 9, 16. [CrossRef] [PubMed]

69. Bogart, L.M.; Ojikutu, B.O.; Tyagi, K.; Klein, D.J.; Mutchler, M.G.; Dong, L.; Lawrence, S.J.; Thomas, D.R.; Kellman, S. COVID-19 Related Medical Mistrust, Health Impacts, and Potential Vaccine Hesitancy Among Black Americans Living with HIV. JAIDS J. Acquir. Immune Defic. Syndr. 2021, 86, 200-207. [CrossRef] [PubMed]

70. Jung, H.; Albarracín, D. Concerns for others increases the likelihood of vaccination against influenza and COVID-19 more in sparsely rather than densely populated areas. Proc. Natl. Acad. Sci. USA 2021, 118, e2007538118. [CrossRef]

71. Largent, E.A.; Persad, G.; Sangenito, S.; Glickman, A.; Boyle, C.; Emanuel, E.J. US Public Attitudes Toward COVID-19 Vaccine Mandates. JAMA Netw. Open 2020, 3, e2033324. [CrossRef]

72. Yin, F.; Wu, Z.; Xia, X.; Ji, M.; Wang, Y.; Hu, Z. Unfolding the Determinants of COVID-19 Vaccine Acceptance in China. J. Med. Internet Res. 2021, 23, e26089. [CrossRef]

73. Robles, A.S.; Gallahue, A.; Bennett, S.; Nerida, T.; Larson, T.; Parker, H.; Riddle, M. Determinants of Covid-19 Vaccine Acceptance among Health Care Providers and Citizens in Nevada. J. Investig. Med. 2021, 69, 235. [CrossRef]

74. Gangarosa, E.; Galazka, A.; Wolfe, C.; Phillips, L.; Miller, E.; Chen, R. Impact of anti-vaccine movements on pertussis control: The untold story. Lancet 1998, 351, 356-361. [CrossRef]

75. Benecke, O.; Deyoung, S.E. Anti-Vaccine Decision-Making and Measles Resurgence in the United States. Glob. Pediatr. Health 2019, 6. [CrossRef]

76. Phadke, V.K.; Bednarczyk, R.A.; Salmon, D.A.; Omer, S.B. Association Between Vaccine Refusal and Vaccine-Preventable Diseases in the United States. JAMA 2016, 315, 1149-1158. [CrossRef]

77. Sallam, M. COVID-19 Vaccine Hesitancy Worldwide: A Concise Systematic Review of Vaccine Acceptance Rates. Vaccines 2021, 9 , 160. [CrossRef] [PubMed] 
78. Roller-Wirnsberger, R.; Lindner, S.; Kolosovski, L.; Platzer, E.; Dovjak, P.; Flick, H.; Tziraki, C.; Illario, M. The role of health determinants in the influenza vaccination uptake among older adults (65+): A scope review. Aging Clin. Exp. Res. 2021, 15, 1-10. [CrossRef]

79. UK Government Scientific Advisory Group for Emergencies. Factors Influencing Covid-19 Vaccine Uptake among Minority Ethnic Groups, 17 December 2020. Available online: https:/ /www.gov.uk/government/publications/factors-influencing-covid19-vaccine-uptake-among-minority-ethnic-groups-17-december-2020 (accessed on 21 March 2021).

80. Santhanes, D.; Wong, C.P.; Yap, Y.Y.; San, S.P.; Chaiyakunapruk, N.; Khan, T.M. Factors involved in human papillomavirus (HPV) vaccine hesitancy among women in the South-East Asian Region (SEAR) and Western Pacific Region (WPR): A scoping review. Hum. Vaccines Immunother. 2018, 14, 124-133. [CrossRef] [PubMed]

81. Ashfield, S.; Donelle, L. Parental Online Information Access and Childhood Vaccination Decisions in North America: A Scoping Review (Preprint). J. Med. Internet Res. 2020, 22, e20002. [CrossRef] 